\title{
Combining Inertially-aided Extended Coherent Integration (Supercorrelation) with 3D-Mapping- Aided GNSS
}

\author{
Paul D Groves, Qiming Zhong \\ University College London, United Kingdom \\ Ramsey Faragher, Paulo Esteves \\ Focal Point Positioning, United Kingdom
}

\begin{abstract}
BIOGRAPHY
Dr Paul Groves is an Associate Professor at University College London (UCL), where he leads a program of research into robust positioning and navigation. He is interested in all aspects of navigation and positioning, including multi-sensor integrated navigation, improving GNSS performance under challenging reception conditions, and novel positioning techniques. He is author of the book Principles of GNSS, Inertial and Multi-Sensor Integrated Navigation Systems. He is the recipient of the 2016 Institute of Navigation Thurlow Award and a Fellow of the Royal Institute of Navigation. He holds a bachelor's degree and doctorate in physics from the University of Oxford. (p.groves@ucl.ac.uk)
\end{abstract}

Qiming Zhong is a PhD student at UCL, specializing in 3D-mapping-aided GNSS. He holds a BSc in Electrical and Electronic Engineering from the University of Nottingham, Ningbo, China and an MSc in Robotics and Computing from UCL.

Dr Ramsey Faragher is the founder and Chief Executive of Focal Point Positioning. He is interested in all aspects of navigation and positioning, including signals of opportunity and advanced GNSS signal processing. He is the recipient of the 2019 Institute of Navigation Per Enge Award and the 2019 GPS World Signals Award, and a Fellow of the Royal Institute of Navigation. In addition, Focal Point Positioning is the recipient of the 2020 Duke of Edinburgh's Navigation Award for Outstanding Technical Achievement. Ramsey holds a bachelor's degree and doctorate in physics from the University of Cambridge.

Dr Paulo Esteves is a Principal Scientist at Focal Point Positioning. He specializes in advanced GNSS signal processing and space systems engineering. He graduated as an Aerospace Engineer from Instituto Superior Técnico, Lisbon, Portugal, in 2007 and received his PhD from the University of Toulouse, France, in 2014.

\begin{abstract}
Conventional GNSS positioning in dense urban areas exhibits errors of tens of meters due to non-line-of-sight (NLOS) reception and multipath interference. Inertially-aided extended coherent integration within the GNSS receiver, as in the case of supercorrelation or S-GPS/GNSS, mitigates these effects by making the receiver more sensitive to directly received signals than reflected signals whenever the receiver is moving. This reduces multipath interference and makes NLOS signals easier to detect using signal-to-noise measurements. 3D-mapping-aided (3DMA) GNSS uses predictions of which signals are NLOS to enhance the positioning algorithms. 3DMA GNSS ranging algorithms can be combined with shadow matching, which uses the signal-tonoise measurements. Both supercorrelation and 3DMA GNSS can improve positioning accuracy in dense urban areas by more than a factor of two. As supercorrelation takes place at the receiver signal processing stage while 3DMA GNSS operates at the positioning algorithm stage, the two techniques are potentially complementary. This paper therefore investigates the benefits of combining them.
\end{abstract}

GNSS signals were recorded using a Racelogic Labsat 3 GNSS front end on a trials van in the Canary Wharf area of London and subsequently processed to generate conventional and supercorrelated ranging and signal-to-noise measurements from the GPS and Galileo satellites. These are then input to several different positioning algorithms, including conventional positioning, shadow matching and likelihood-based 3DMA ranging and a combination of shadow matching and likelihood-based 3DMA ranging.

Single-epoch positioning results using code-only pseudo-ranges clearly demonstrate the benefit of supercorrelation with position errors using S-GNSS measurements $40 \%$ smaller than those using conventional least-squares positioning techniques. 3DMA 
GNSS improves the position accuracy by about $25 \%$ in the denser environments, but does not bring any benefits in the more open areas. When supercorrelation is combined with filtered carrier-smoothed pseudo-ranges using outlier detection to reject code components that inconsistent with the carrier-based component, the least-squares position errors are reduced by a factor of 5.7 in the densest of environments and a factor of 3.5 elsewhere. With these filtered measurements, 3DMA GNSS techniques have little impact on the positioning accuracy. Thus, 3DMA GNSS techniques are likely to be more useful for snapshot positioning techniques and potentially for the initialization of filtered solutions than for continuous positioning.

\section{INTRODUCTION}

Conventional GNSS positioning in dense urban areas can exhibit errors of tens of meters, sometimes more than a hundred meters. The two main causes of this are non-line-of-sight (NLOS) reception and multipath interference [1]. NLOS reception is due to complete blockage of the direct line-of-sight (LOS) GNSS signals by tall buildings, other structures (e.g. walls), or passing vehicles. Signals are then often received via reflected paths leading to a ranging error equal to the extra path delay, i.e. the difference between the reflected and direct paths. Multipath interference is due to reception of signals via multiple paths from the satellite to the receiver, including the direct path. Ranging errors due to multipath interference can be positive or negative and depend on the relative amplitude of the reflected signal(s), their path delay and the receiver design. Metallized glass is a much stronger reflector than brick and stone. NLOS reception and multipath interference are often grouped together and referred to simply as "multipath". However, to do so is highly misleading as the two phenomena have different characteristics and can require different mitigation techniques. There are several different strategies to mitigate these vulnerabilities [2]. Here, the synergy between super-correlation, or S-GPS/GNSS and 3D-mapping-aided (3DMA) GNSS will be investigated. Operating at different stages of the GNSS processing chain, the two techniques offer distinct enhancements in key performance parameters of GNSS receivers.

S-GNSS operates at the receiver signal processing stage, enabling extended coherent integration by using a low-cost inertial measurement unit (IMU) to measure the antenna motion, which is then corrected for. Compared with noncoherent integration, this increases the receiver sensitivity, enabling it to operate at lower signal-to-noise levels. Extended coherent integration also makes the receiver more sensitive to changes in Doppler shift. Consequently, when the receiver is moving, reflected signal components, which experience a different Doppler shift from their direct counterparts, can be attenuated. Thus, the antenna's effective gain pattern becomes directional and is different for each signal tracked. Attenuating reflected signals reduces the ranging errors due to multipath interference and makes it easier to distinguish NLOS signals from LOS by their signal-to-noise levels. S-GNSS is described further in Section 2.

3DMA GNSS operates at the subsequent navigation solution computation stage. In conventional GNSS positioning algorithms, all signals are assumed to follow a direct path from the satellite to the user equipment antenna, which leads to errors when signals are received by a reflected path. In 3DMA GNSS, 3D building mapping is used to predict which signals are directly visible and which are blocked by buildings at any given location. In some implementations, path delays are also predicted. The positioning algorithms are then modified to take this information into account. There are many different ways of implementing 3DMA GNSS and individual algorithms may be configured in different ways. This leads to a three way trade-off between the positioning accuracy, the size of initial position uncertainty that can be handled and the computational load. The two main approaches are shadow matching, which determines position by comparing measured and predicted satellite visibility, and ranging-based positioning algorithms; these may be combined. Section 3 reviews the different approaches to 3DMA GNSS and summarizes the positioning algorithms assessed here. A full description of the algorithms is presented in Appendix A.

GNSS signals were recorded using a Racelogic Labsat 3 GNSS front end in the Canary Wharf area of London, which contains many tall glass-covered buildings similar to those found in North American and Asian cities. This data was subsequently processed to generate conventional and S-GNSS ranging and signal-to-noise measurements from the GPS and Galileo satellites. These were then processed using different position algorithms. Section 4 presents a comparison of single-epoch conventional and 3DMA GNSS position solutions using both conventional and S-GNSS ranging measurements, followed by a comparison of single-epoch and filtered conventional positioning solutions using the S-GNSS measurements. Section 5 presents conclusions and Section 6 recommends topics for future work.

Applications that could benefit from this work include situation awareness of emergency, security and military personnel; emergency caller location for both people and vehicles; navigation for the visually impaired; visitor guidance through cities; tracking vulnerable people and valuable assets; location-based charging; mapping environmental features; enforcement of curfews, restraining orders and other court orders; intelligent transport systems and autonomous vehicles; location-based services; mobile gaming and augmented reality. 


\section{SGPS/GNSS}

The Focal Point Positioning S-GPS/S-GNSS technology [3][4] is a suite of methods, protected by patents, that provide softwarebased improvements to GNSS receivers. All methods build upon a core technology called supercorrelation. Further techniques such as ultracorrelation and SkyScan build upon this core technique [5].

The core S-GNSS technology, supercorrelation, provides improvements to the delay lock loop (DLL), frequency lock loop (FLL) and phase lock loop (PLL) control feedback stages of a GNSS receiver [6][7] by extending the coherent integration time. Coherent integration is where the in-phase $(I)$ and quadraphase $(Q)$ correlator outputs within the receiver are summed separately, preserving the phase information, while noncoherent integration sums $I^{2}+Q^{2}$, destroying the phase. The DLL used for code tracking performs noncoherent integration implicitly. For coherent integration, the post-correlation signal-to-noise ratio increases in proportion to the number of samples accumulated, whereas for noncoherent integration the signal-to-noise ratio is only proportional to the square root of the number of samples.

Current generation GNSS receivers perform a small amount of coherent integration, typically less than 20 milliseconds, and then noncoherently integrate to boost acquisition sensitivity if required. The reasons for this $\sim 20 \mathrm{~ms}$ limit are historical limitations of data modulation bits, crystal oscillator stability, and receiver motion during the integration period. S-GNSS overcomes these limitations to provide over a second of coherent integration, while undergoing complex motions, on low cost platforms. The benefit is high sensitivity coupled with unique multipath mitigation capabilities providing a high accuracy and high integrity solution for positioning in traditionally difficult environments, such as dense urban areas.

A major problem with using noncoherent integration instead of coherent integration is the susceptibility to multipath interference. Noncoherent integration destroys the phase information stored within the captured data before combining it, resulting in LOS and NLOS signals accumulating within the same correlation peak, even if their Doppler shifts are different. This distorts the correlation function, leading to erroneous code phase estimates, which in turn leads to erroneous positions estimates [8][9][10]. Supercorrelation employs motion compensation of the correlation process itself to separate out in the frequency domain the energy from different incoming directions, allowing the LOS energy to be isolated and allowing a LOS-only correlation peak to be formed, regardless of the number of incoming reflected signals at the same moment that would normally cause distortion of the desired correlation peak

\subsection{Building a Supercorrelator}

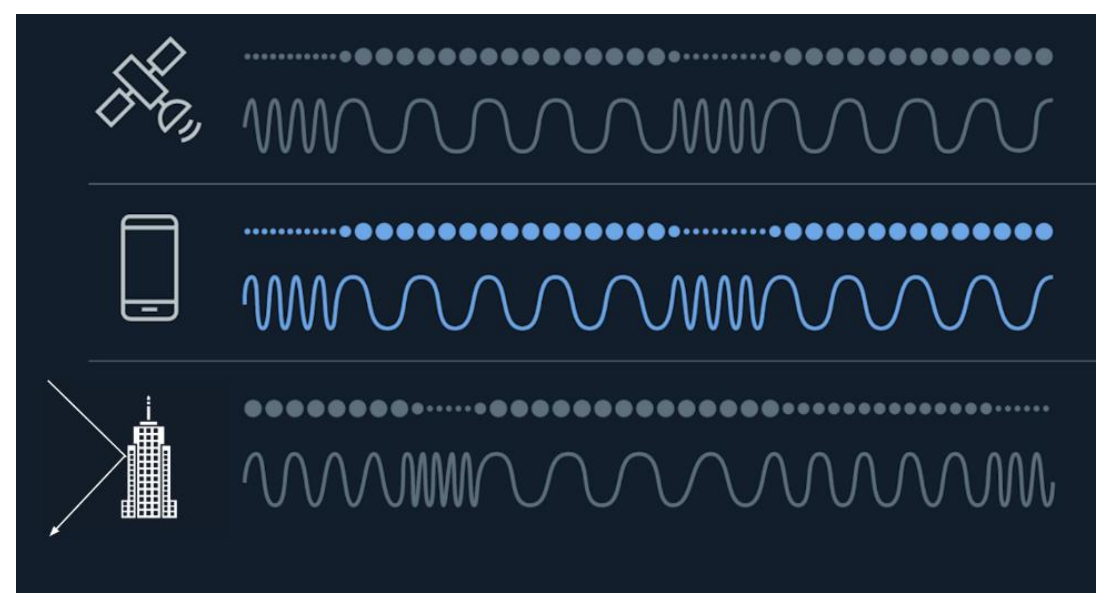

Figure 1: As the velocity of the receiver changes, the Doppler shift of the directly received signal changes (top). Supercorrelation enables the receiver-generated replica signal that is correlated with the incoming signal to match this change (middle). Reflected signals in the local environment suffer different Doppler variations than the desired line-of-sight signal (bottom). This means that the supercorrelator that is created for a given satellite broadcast couples strongly to the desired line of sight version of the signal, but attenuates any reflected signals arriving from different directions. (Figure reproduced from [5])

Coherent integration requires GNSS data to be combined with both perfect code-phase alignment, and perfect carrier-phase alignment. The latter is traditionally challenging due to the level of knowledge required of the non-linear variations of the user clock and user motion during the integration period. Inertial processing is required to reconstruct non-linear motion of the GNSS antenna and non-linear oscillator variations must also be modelled correctly. The degree of multipath mitigation provided 
increases with both the speed of the receiver through space (relative to the reflecting surfaces in the environment) and the coherent integration time. Figure 1 illustrates this.

Coherent integration has traditionally been limited by the navigation data message bits that are modulated onto all of the legacy GNSS signals. In order to provide extended coherent integration times beyond this, either modern pilot (i.e., data-free) signals should be used, or the navigation bits must be stripped from the data stream. The latter requires either onboard prediction of the data bits based on previous messages or an external assistance stream containing the data message. It is possible to strip the navigation bits through a signal squaring method that involves multiplying the complex sample by itself rather than by its complex conjugate. This squares the signal but maintains a complex phase in the output signal which is free of BPSK modulation.

To build a supercorrelator, the component of the receiver motion in the direction of interest is used to produce a motioncompensation phasor sequence. A phasor sequence corresponding to the estimates of the local oscillator variations is also constructed. When these phasors are added to the incoming radio data they align the phases of successive GNSS data such that they can be summed in phase, with motion and clock errors compensated. The complex phasors can be applied directly to the incoming samples from the analog-to-digital converter (ADC), or can be applied directly to the receiver-generated pseudorandom-noise (PRN) sequence in the correlator bank. A third option is to maintain the existing front-end architecture of a receiver and to collect the complex correlator taps from the correlation engine (the early set, late set, and prompt correlator outputs that are passed to the DLL and PLL to track code phase and carrier phase) and to apply the supercorrelator phasor to these taps.

Whichever method is implemented, the result is that the correlator output taps have been rotated by the supercorrelator phasor to a new set of angles before they are coherently integrated. The result is a correlation peak corresponding only to the energy that has arrived from a selected direction. This beamforming technique is sometimes referred to as synthetic aperture GNSS [11][12]. Supercorrelation is an extension of synthetic aperture processing that simultaneously solves for non-linear motion and non-linear oscillator errors via a probabilistic joint estimator that processes data all of the satellites simultaneously to estimate motion and clock states. This is an important step beyond traditional synthetic aperture processing (which does not solve for high order oscillator error states), and is required when using the very low quality oscillators such as those found in low-cost consumer GNSS receivers and smartphones.

The selected directions for motion compensation are typically the expected directions to each given satellite in the sky, but any direction can be selected. Thus, a reflected signal component may be selected, which can be useful where 3D mapping can be used to predict the path delay. When a bank of supercorrelators is built for a given measurement epoch in order to sky the full range of azimuth and elevation of the sky above the receiver the resulting data are referred to as Skyscans and can be imaged as shown in Figure 2. The angles of arrival of LOS and NLOS signals from each satellite can therefore be directly measured.
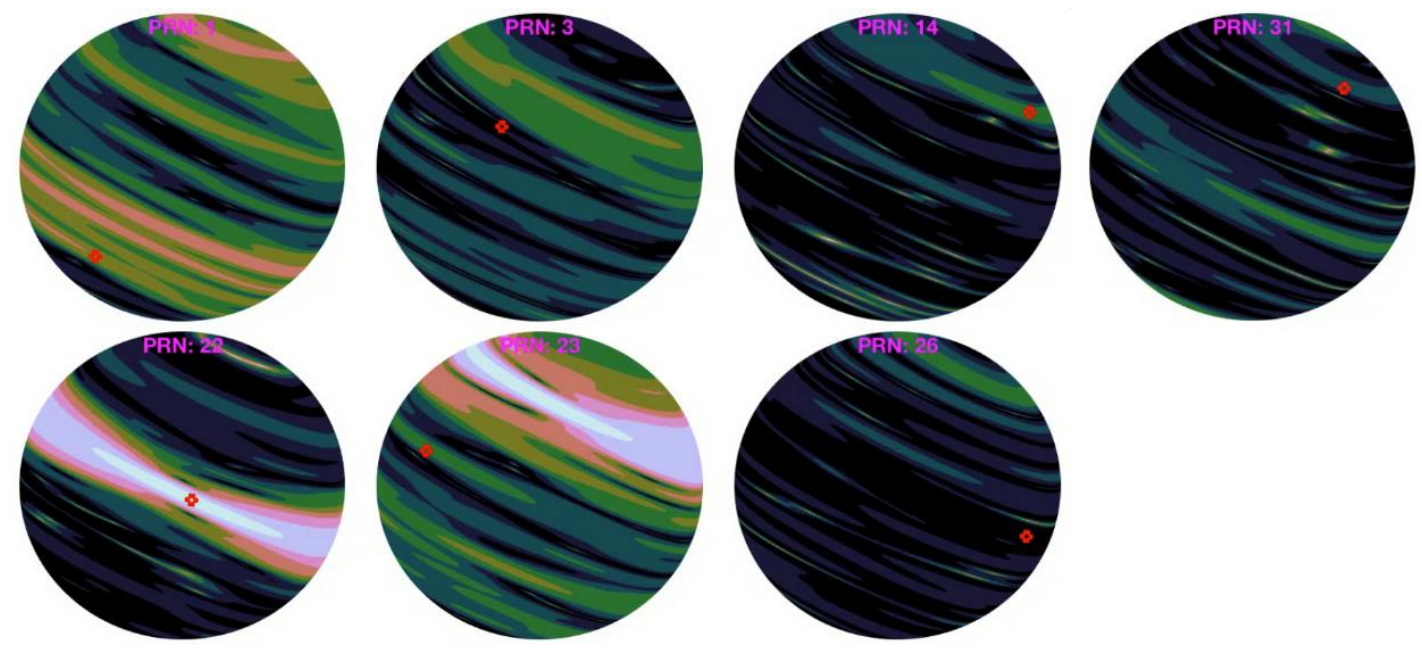

Figure 2: Skycan outputs for 7 GPS satellites a San Francisco urban canyon running from North West to South East. Each disc is an azimuth-elevation plot of the sky, with a red mark showing the position of a given satellite (the PRN number is given in each title). The colours in the plots represent the energy being detected from all azimuths and elevations for each satellite. PRN 22 for example is a strong overhead satellite. PRN 23 however is exhibiting a strong non-line-of-sight signal on the North side of the receiver at moderate elevation, whereas only a weak signal is being detected from the true satellite direction in the West at moderate elevation. No line-of-sight energy is detectable at all for PRN 26, although there are more than three weak versions of the signal being received from the Northern side at various elevations. (Reproduced from [5]) 
SkyScan technology uses a bank of supercorrelators to produce a skyplot of received signal strength as a function of azimuth and elevation. This can be used to determine the strengths and angles of arrival reflected signal components as well as the desired line of sight signal. Figure 2 shows some examples.

\subsection{Performance Benefits}

Some of the performance benefits of S-GPS/GNSS may be illustrated using GPS data collected from a vehicle test in London during 2016. Figure 3(a) shows the measured $C / N_{0}$ of one of the GPS satellites with S-GPS on and off. Satellite visibility predictions obtained using 3D mapping and the truth reference are also shown. It can be seen that the $C / N_{0}$ during NLOS reception is lower when S-GPS processing is on, demonstrating that S-GNSS can attenuate reflected signals. Figure 3(b) then presents the Cumulative distribution function (CDF) of the same $C / N_{0}$ data,showing a slight shift towards lower $C / N_{0}$ values when GPS is on.

(a)

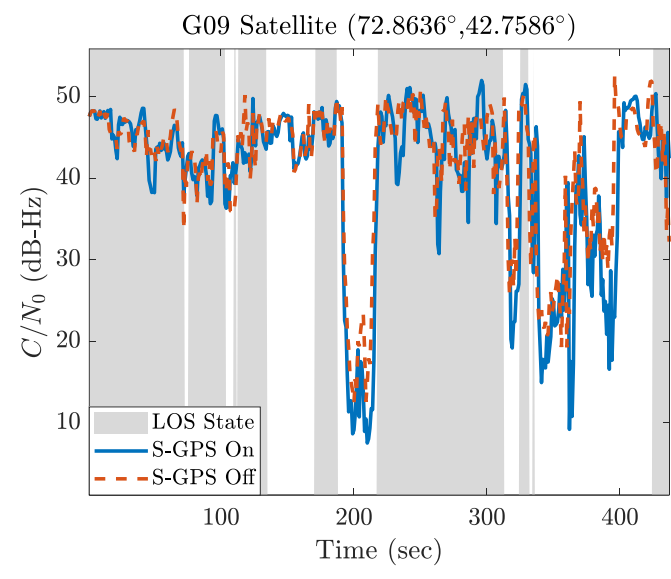

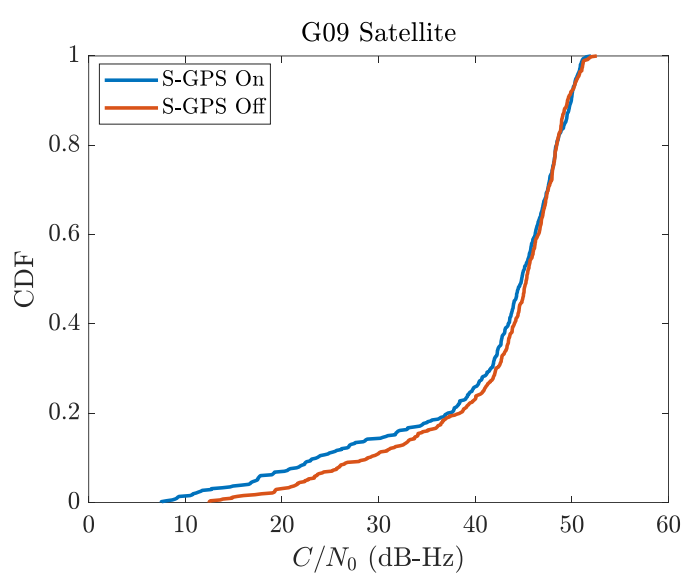

(b)
Figure 3: (a) $C / N_{0}$ time series for GPS PRN09 with S-GPS on and off, overlaid to satellite's visibility predicted (grey = LOS; white $=$ NLOS) and (b) Cumulative distribution function of the measured $C / N_{0}$ for GPS PRN09, both obtained from test trials in Canary Wharf area, London

Figure 4 shows the carrier-power-to-noise-density ratio, $C / N_{0}$, measurement distributions for LOS and NLOS signals from all of the GPS satellites with and without supercorrelation. Again, the truth reference and 3D mapping were used to determine which signals were NLOS and which were direct LOS. With supercorrelation on, there is less overlap between the LOS and NLOS distributions. This makes it easier to infer from the $C / N_{0}$ measurements whether a signal is LOS or NLOS. Thus, GNSS shadow matching can be expected to perform better with supercorrelated measurements than with the corresponding conventional measurements. The same applies to conventional GNSS positioning algorithms that use $C / N_{0}$ to weight the measurements.
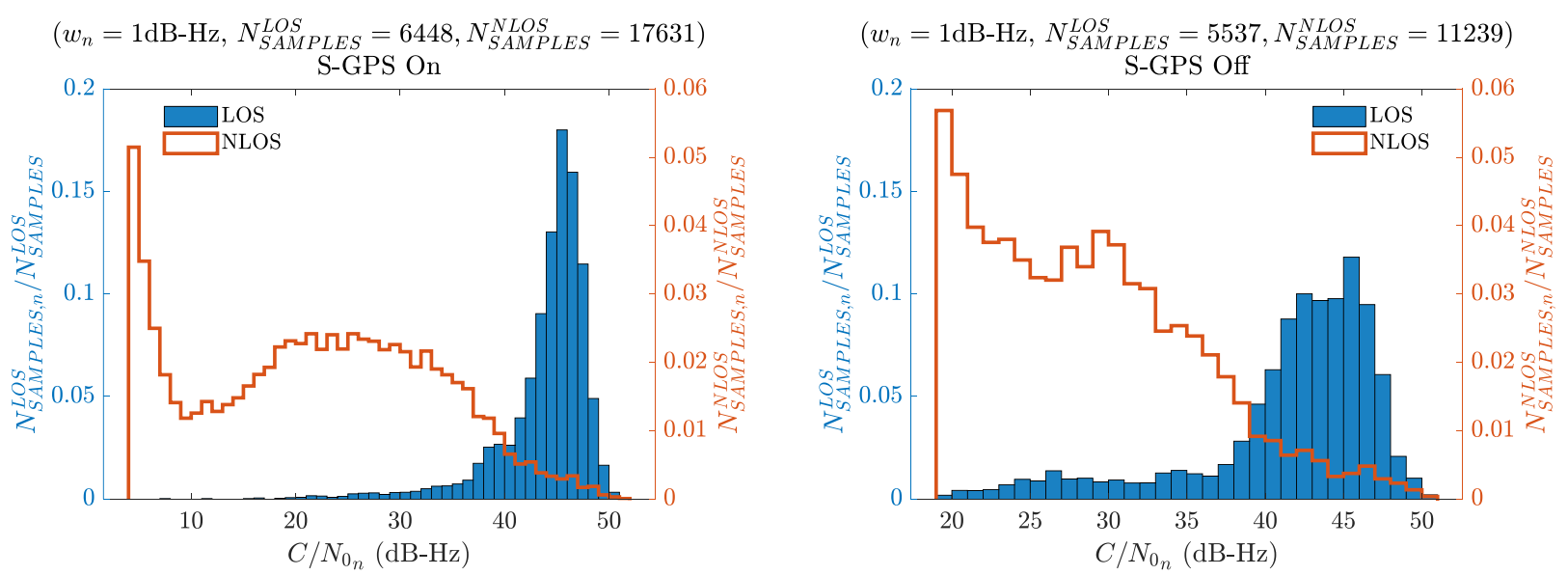

Figure 4: $C / N_{0}$ histograms for LOS/NLOS values when S-GPS is on (left) and off (right), for all tracked satellites using data collected in the city of London. 
Figure 5 shows the pseudo-range residuals of a conventional least-squares positioning algorithm using LOS and NLOS signals from the GPS satellites with and without supercorrelation. The standard deviation for both the LOS and NLOS signals is much smaller using the superorrelated measurements than the conventional measurements. Thus, any ranging-based GNSS positioning algorithm, whether aided by 3D mapping or not, can be expected to perform better with supercorrelated measurements than with the corresponding conventional measurements.
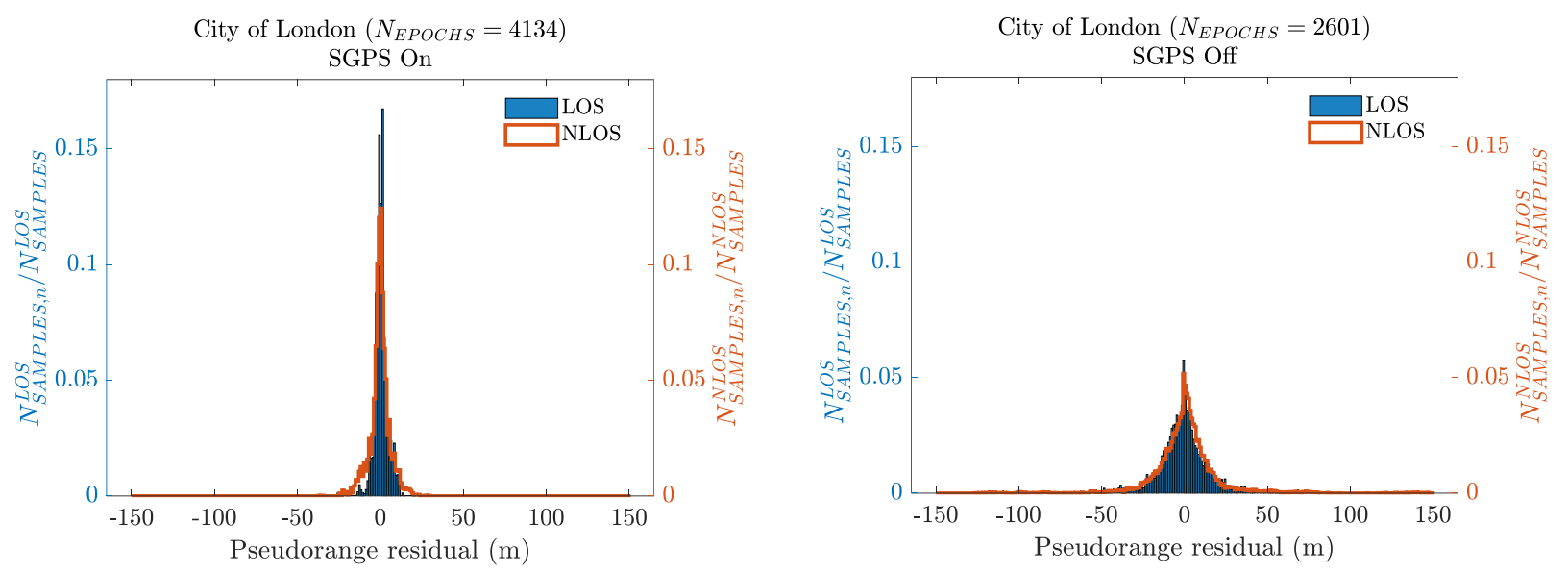

Figure 5: Empirical distributions of LOS and NLOS pseudo-range residuals when S-GPS is on (left) and off (right) using data collected in the city of London.

\section{3D-MAPPING-AIDED GNSS}

Over the past decade, there has been a lot of interest in 3D-mapping-aided (3DMA) GNSS. The simplest approach, which originally dates from the 1980s when there were only a few satellites, is terrain height aiding [13]. This uses a digital terrain model (DTM), also known as a digital elevation model (DEM) to constrain the position solution to the terrain surface. More recent 3DMA GNSS technique improves performance in dense urban areas by using maps of the buildings to predict which signals are directly visible at any given location. Many different approaches have been demonstrated, but each may be broadly classified as either ranging or shadow matching. The simplest 3DMA ranging algorithms use predicted satellite visibility from 3D mapping to discard or downweight those signals predicted to be NLOS at a known location [14][15]. More sophisticated approaches use LOS/NLOS predictions over a range of candidate positions, reflecting the uncertainty of initialization from conventional GNSS positioning in a dense urban environment [16]. Some researchers use the 3D mapping to correct those signals predicted to be non-line-of-sight (NLOS) [17][18][19]. This should enable higher accuracy, but computing NLOS corrections using 3D mapping is more computationally intensive than just predicting satellite visibility, limiting the number of candidate positions that can be considered if corrections are to be computed in real-time. In [20], precomputing NLOS corrections over a grid of candidate positions, satellite azimuths and elevations is proposed, which would require around 10GB of data per $\mathrm{km}^{2}$. Alternatively, the processing load can be reduced by using predicted pseudo-ranges based on the satellite elevation angle and distance to the reflecting surface [21][22]. 3DMA ranging algorithms have also been applied to the components of multipathcontaminated signals, separated using Doppler-domain signal processing when the receiver is moving [23].

Shadow matching determines position by comparing the measured signal availability, carrier-power-to-noise-density ratio, $C / N_{0}$, or signal to noise ratio (SNR) measurements with satellite visibility predictions from $3 \mathrm{D}$ mapping over a grid of candidate positions [24][25][26][27][29][30][31]. Shadow matching tends to be more accurate in the across-street direction due to the building geometry. Conversely, ranging tends to be more accurate in the along-street direction because there are more direct LOS signals in this direction. Thus, in general, best performance is obtained by using both 3DMA ranging and shadow matching $[16]$.

As well as the algorithm design, 3DMA GNSS performance is also affected by the receiver design, the environment, and the 3D mapping quality. For both 3DMA GNSS and conventional GNSS, geodetic receivers give better performance than consumergrade receivers, which in turn give better performance than smartphones, mainly due to the polarization discrimination of the antenna. Environmental factors that impact performance include sky visibility, building height and street width, building materials and passing vehicles. All positioning methods exhibit poorer accuracy in environments dominated by glass and steel buildings, as these buildings produce stronger reflected GNSS signals, increasing multipath errors and making it more difficult to distinguish LOS and NLOS signals using $C / N_{0}$. In terms of 3D mapping quality, using full 3D city models (level of detail 2) can be expected to give better performance than simple block models that do not account for variations in building cross section 
with height (level of detail 1). Out-of-date mapping is also a problem. The impact of these factors on shadow matching is further discussed in [32].

Many of the applications that benefit from 3DMA GNSS can run on a smartphone. A smartphone platform also provides a convenient means of downloading building boundary or 3D mapping data from a server, a potential augmentation to assisted GNSS [7]. The Android application program interface (API) now provides "raw GNSS measurements" in the form of pseudorange and $C / N_{0}$ measurements from compatible GNSS chipsets, enabling advanced GNSS positioning algorithms to be hosted on the device's CPU [34]. Thus, 3DMA GNSS algorithms can be implemented in real-time on an Android smartphone, as has already been demonstrated for shadow matching [35]. Alternatively, the GNSS measurements may be uploaded to a server for remote computation of the position solution [31].

\subsection{UCL's 3DMA GNSS Positioning Algorithms}

At UCL, the focus has been on 3DMA GNSS algorithms that can potentially operate in real-time over a wide search area, handling a large initialization uncertainty. Therefore, satellite visibility is predicted using pre-computed building boundaries [33] for a grid of candidate positions. These describe the minimum elevation above which satellite signals can be received at a series of azimuths and are precomputed for each candidate position. A signal can then be classified as LOS or NLOS simply by comparing the satellite elevation with that of the building boundary at the corresponding azimuth. Note that data storage requirements for precomputed building boundaries are about 100 times less than for precomputed NLOS corrections.

UCL's implementation of 3DMA GNSS comprises five stages, as shown in Figure 6. A terrain-height-aided least-squares ranging (LSR) algorithm with outlier detection is used for initialization [36]. The residuals are then used to determine the size of the search area for the subsequent stages. Next, a likelihood-based ranging (LBR) algorithm scores candidate position hypotheses according to the correspondence between measured and predicted pseudo-ranges. Different error distributions are assumed at each candidate position according to which signals are predicted to be NLOS at that location [37]. A shadow matching (SM) solution [29] is computed and then integrated with the LBR solution in the hypothesis domain before extracting a position solution from the combined likelihood surface. Further details follow. An alternative approach based on extracting multiple position hypotheses from the LBR and SM distributions and integrating the best matching hypotheses has also been investigated, but is not presented here. Currently, only single-epoch 3DMA positioning has been implemented.

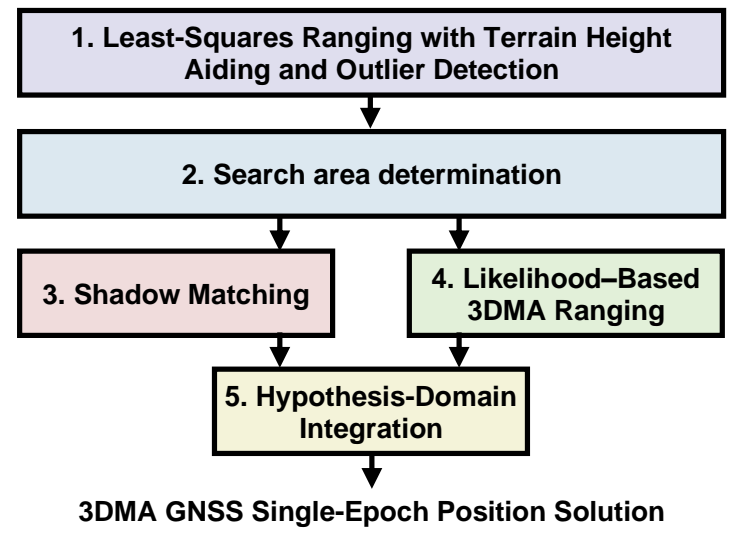

Figure 6: Single-Epoch 3D-mapping-aided GNSS algorithm components

UCL 3DMA GNSS results published between 2016 and 2019 have been found to be unreliable, so the relevant publications are not cited here. These papers may eventually be withdrawn. The results presented here should therefore be treated as the first published results from UCL of the LBR and hypothesis-domain integration algorithms.

The least-squares ranging algorithm weights the pseudo-range measurements according to the corresponding $C / N_{0}$ measurements. Inter-constellation timing bias corrections from the navigation data messages are applied. Outliers are removed with a sequential elimination loop. If the detection threshold is exceeded, the measurement with the largest residual is eliminated and the solution re-calculated. This continues until either the threshold is met or only four measurements remain.

Once an initial position solution has been obtained using pseudo-ranges only, the terrain height at the corresponding position is obtained from a digital terrain model (DTM), also known as a digital elevation model (DEM). This is used to generate an additional virtual range measurement, comprising the distance from the center of the Earth to the user antenna [38]. The LSR 
algorithm then computes a terrain-height-aided position solution using the pseudo-range and virtual range measurements. An incorrect horizontal position solution leads to an incorrect terrain height, so the terrain height determination and LSR positioning algorithms are iterated up to 20 times to minimize these errors. Full details are given in Appendix A.1.

The next step is to determine the search area for 3DMA GNSS as both the likelihood-based ranging and shadow matching algorithms score candidate positions according to the correspondence between measurements and predictions. If the search area is too small, it will not include the true position, making a correct solution impossible. Conversely, if the search area is too large, there may be multiple locations where there is a good match between measurements and predictions. Such ambiguous solutions can be a common feature of shadow matching in particular due to repeating building geometry. Therefore, the search radius is scaled according to the quality of the LSR solution, which is estimated from the residuals. Once the search area has been determined, a regular grid of candidate positions is set up with spacing proportional to the search area to maintain a constant processing load. The DTM is used to associate a terrain height with each horizontal position, removing the need for a threedimensional search space. Terrain-height aiding is thus implicit in 3DMA GNSS. Full details are given in Appendix A.2.

Shadow matching comprises the following steps [29]:

- For each candidate position in the search area, the satellite visibility is predicted using the building boundaries precomputed from the 3D city model.

- The probability that each received signal is direct LOS is determined from the $C / N_{0}$ measurements using an appropriate statistical model.

- Each candidate position and satellite is scored according to the match between the predicted and measured satellite visibility; visibility predictions are no assumed to be perfect

- An overall likelihood score is computed for each position by multiplying the scores for each satellite.

Full details are given in Appendix A.3.

The likelihood-based 3DMA ranging algorithm, comprising the following steps [37]:

- For each candidate position, the satellite visibility is predicted using the building boundaries.

- At each candidate position, one of the satellites predicted to be direct LOS is selected as the reference.

- At each candidate position, the direct LOS range to each satellite is computed and then measurement innovations are calculated by subtracting the computed ranges from the measured pseudo-ranges and then differencing with respect to the reference satellite. This eliminates the receiver clock offset.

- At each candidate position, the measurement innovation for each satellite predicted to be NLOS is re-mapped by using a skew normal distribution to determine the cumulative probability and then substituting the corresponding direct LOS innovation with the same cumulative probability. This enables the NLOS measurements to be scored using an asymmetric distribution which accounts for the error due to NLOS reception always being positive, but treated like Gaussian-distributed measurements in the next step.

- A joint likelihood score for each candidate position is computed using the measurement innovations and their error covariance matrix, noting that all innovations are correlated with each other due to the common reference satellite.

Full details are given in Appendix A.4.

Finally, the likelihood-based ranging and shadow matching solutions are combined. This exploits the fact that shadow matching tends to be more accurate in the cross-street direction in dense urban areas, while ranging based position solutions tend to be more accurate in the along-street direction. Hypothesis-domain integration is used, which combines the shadow-matching and 3DMA ranging scores to give a single score for each candidate position. This down-weights any parts of the search area that are given a high score by shadow matching and a low score by likelihood-based ranging, or vice versa. Finally, the position solution is obtained by using the combined scores to weight the candidate positions. Full details are given in Appendix A.5.

\section{EXPERIMENTAL TESTS}

GNSS signals were recorded using a Racelogic Labsat 3 GNSS front end on a trials van in the Canary Wharf area of London and subsequently processed to generate conventional and supercorrelated ranging and signal-to-noise measurements from the GPS and Galileo satellites. A truth reference was obtained from a Novatel iMAR INS/GNSS system [39]. Figure 7 shows the van trajectory from the truth reference data and Figure 8 shows the equipment. The van travelled clockwise on the route and the northmost section was traversed twice. The central area, highlighted in red, contains many tall buildings (the highest is $235 \mathrm{~m}$ ) clad in metalized glass and steel, which presents a very challenging reception environment for GNSS. Such buildings are common in North American and Asian cities. The rest of the route is much lower density and is typical of European cities outside the central business district. Separate positioning performance statistics are provided for the central area and the other areas. 


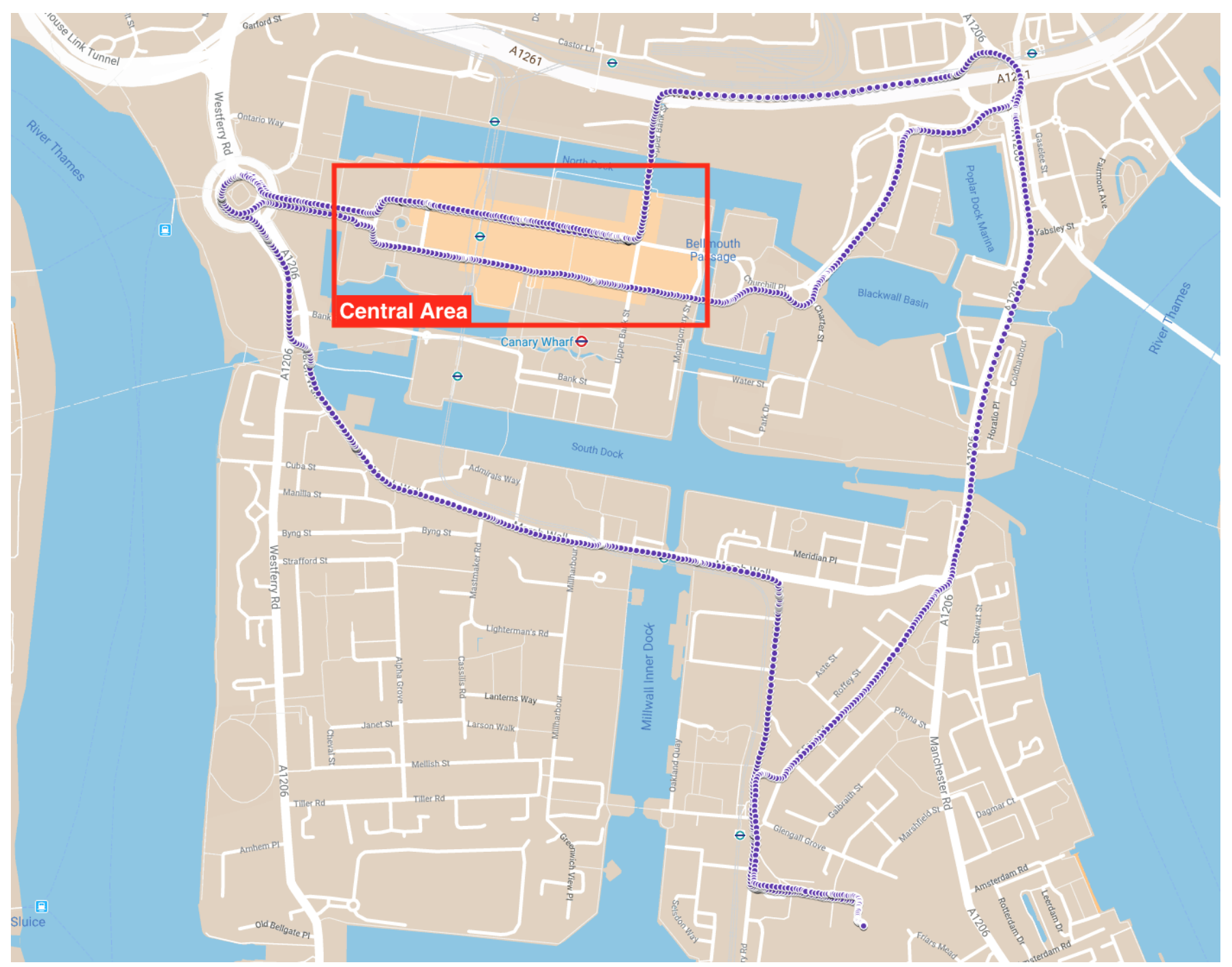

Figure 7: True trajectory of van trial in Canary Wharf, London. Background map @ Google.

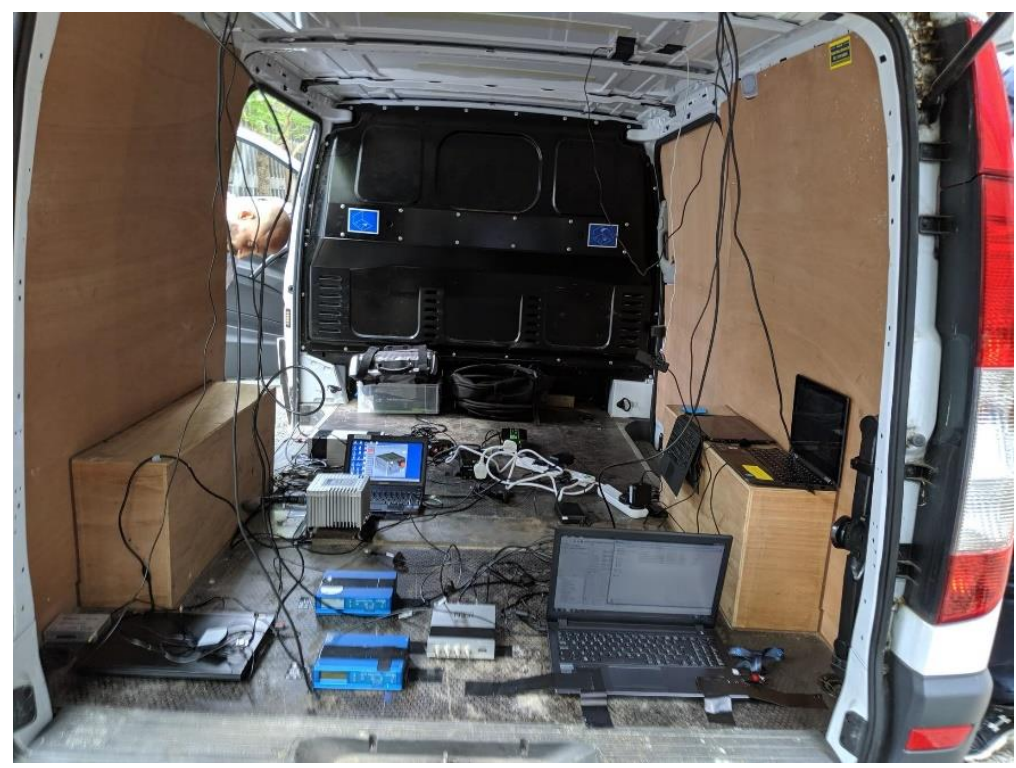

Figure 8: Test equipment in the back of the trials van. 
Using the positioning algorithms described in Appendix A and summarized in Section 3.1, four position solutions were computed with both the conventional and S-GNSS measurements. These are a conventional least-squares ranging (LSR) solution with terrain-height aiding and outlier detection, and three 3DMA GNSS solutions: shadow matching (SM), likelihood-based ranging (LBR) and hypothesis domain integration (HDI) of the SM and LBR solutions. Note that terrain-height aiding is implicit in 3DMA GNSS. Two different types of S-GNSS pseudo-range measurements were used, simple code-based pseudo-ranges and filtered carrier-smoothed pseudo-ranges with outlier detection applied to reject the code component where it is inconsistent with the carrier-based component. This can correct NLOS reception errors in cases where the reflected signal has the same Doppler shift as the direct, such as where the reflecting surface is parallel to the direction of travel. Carrier-smoothing also significantly reduces multipath errors when the multipath environment is rapidly changing. The basic S-GNSS measurements are representative of a one-time position fix while the filtered measurements are representative of continuous positioning.

Table 1 presents horizontal radial position error statistics for all 12 solutions. Figures 9, 10, 11, 12, 13 and 14 show the position error as a function of time and the position error distribution for the Central and other areas for, respectively, the LSR solution using conventional measurements, the HDI solution using conventional measurements, the LSR solution using S-GNSS measurements, the HDI solution using S-GNSS measurements, the LSR solution using filtered S-GNSS measurements and the HDI solution using filtered S-GNSS measurements.

The results show a large improvement from using supercorrelation. With conventional least-squares positioning, using S-GNSS reduces the root mean square (RMS) position error by $39 \%$, both in the central high-density area and elsewhere. The smaller impact in the central area is likely due to the limited number of direct LOS signals as supercorrelation has more impact on multipath interference than NLOS reception. Using the filtered pseudo-range measurements reduces the RMS position error by a further factor of 3.5 in the central high-density area and a factor of 2.1 elsewhere.

Comparing the 3DMA GNSS solutions with the LSR solutions, it can be seen that a shadow-matching-only solution does not improve the position accuracy. This is to be expected as shadow matching was always intended to be used alongside rangingbased positioning, primarily to improve accuracy in the cross-street direction. With conventional GNSS measurements, the RMS position error of the LBR solution generally performs slightly better than the LSR solution in the central area, but performs worse in the other areas. However, the HDI solution performs consistently better than LSR, with a $27 \%$ reduction in RMS position error in the central area and a $9 \%$ reduction elsewhere. This demonstrates the synergy of shadow-matching with ranging. With basic S-GNSS, the benefits of 3DMA GNSS are smaller with the LBR solution consistently worse than the LSR solution and the HDI solution reducing the RMS position error by $23 \%$, compared with LSR, in the central area, but increasing it elsewhere.

Using the filtered S-GNSS pseudo-ranges, the LBR solution performs consistently worse than the LSR solution, while the HDI solution is of a similar accuracy to the LSR solution. Because the filtering of the measurements already reduces the impact of NLOS reception, there is much less scope for 3DMA GNSS techniques to improve position accuracy.

Table 1: Horizontal radial position error statistics for single-epoch positioning in Canary Wharf

\begin{tabular}{|c|c|c|c|c|c|c|c|c|c|c|c|c|}
\hline Measurements & \multicolumn{4}{|c|}{ Conventional } & \multicolumn{4}{|c|}{ Basic S-GNSS } & \multicolumn{4}{|c|}{ Filtered S-GNSS } \\
\hline Positioning algorithm & LSR & SM & LBR & HDI & LSR & SM & LBR & HDI & LSR & SM & LBR & HDI \\
\hline Root mean square (RMS) error, $\mathrm{m}$ & 47.9 & 53.1 & 47.6 & 36.1 & 29.1 & 37.4 & 32.1 & 26.4 & 9.2 & 11.3 & 10.4 & 9.2 \\
\hline $\begin{array}{l}\text { Mean absolute deviation (MAD) } \\
\text { error, } \mathrm{m}\end{array}$ & 20.6 & 27.3 & 24.9 & 14.3 & 14.8 & 20.7 & 18.0 & 14.9 & 5.7 & 8.5 & 6.4 & 5.7 \\
\hline Central area RMS error, $\mathrm{m}$ & 85.3 & 93.2 & 76.9 & 62.2 & 52.0 & 66.1 & 54.9 & 39.9 & 14.9 & 17.4 & 15.9 & 14.6 \\
\hline Central area MAD error, $\mathrm{m}$ & 52.2 & 64.1 & 49.9 & 31.5 & 36.3 & 47.7 & 37.0 & 24.7 & 9.0 & 13.4 & 10.6 & 9.3 \\
\hline Central area minimum error, $\mathrm{m}$ & 1.2 & 0.8 & 0.2 & 0.6 & 0.4 & 0.3 & 0.3 & 0.6 & 0.4 & 0.9 & 0.4 & 0.5 \\
\hline Central area maximum error, $\mathrm{m}$ & 506.6 & 504.3 & 516.7 & 403.4 & 231.1 & 227.2 & 187.3 & 240.9 & 138.9 & 115.3 & 116 & 119.9 \\
\hline Other areas RMS error, $\mathrm{m}$ & 19.7 & 24.0 & 29.5 & 17.9 & 12.0 & 16.6 & 16.7 & 18.9 & 5.6 & 7.7 & 7.2 & 6.0 \\
\hline Other areas MAD error, $\mathrm{m}$ & 8.5 & 13.2 & 15.3 & 7.8 & 6.7 & 10.6 & 10.8 & 11.2 & 4.4 & 6.6 & 4.8 & 4.3 \\
\hline Other areas minimum error, $\mathrm{m}$ & 0.2 & 0.3 & 0.3 & 0.2 & 0.1 & 0.3 & 0.1 & 0.1 & 0.1 & 0.3 & 0.2 & 0.2 \\
\hline Other areas maximum error, $\mathrm{m}$ & 174.9 & 173.6 & 203.3 & 190.8 & 97.1 & 109.4 & 158.6 & 155.6 & 39.8 & 29.9 & 53.4 & 54.7 \\
\hline
\end{tabular}



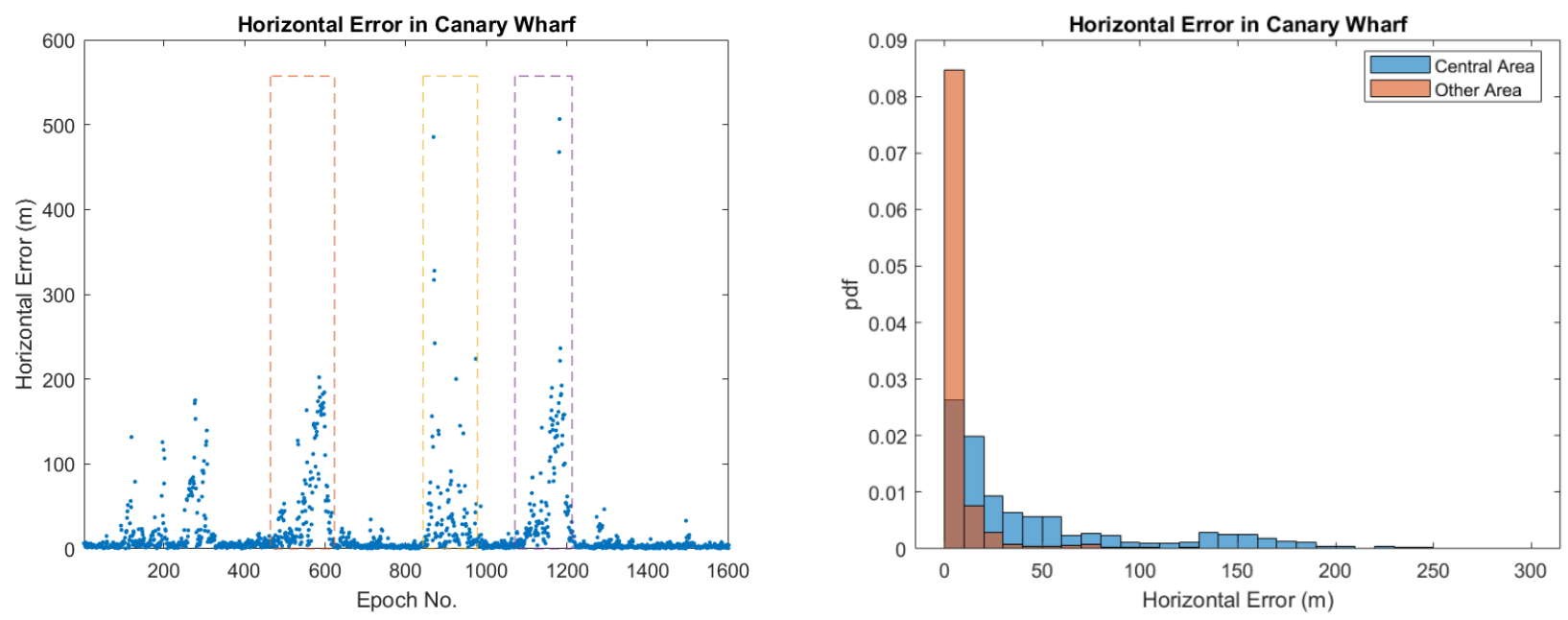

Figure 9: Horizontal radial position error using least-squares ranging with conventional GNSS measurements
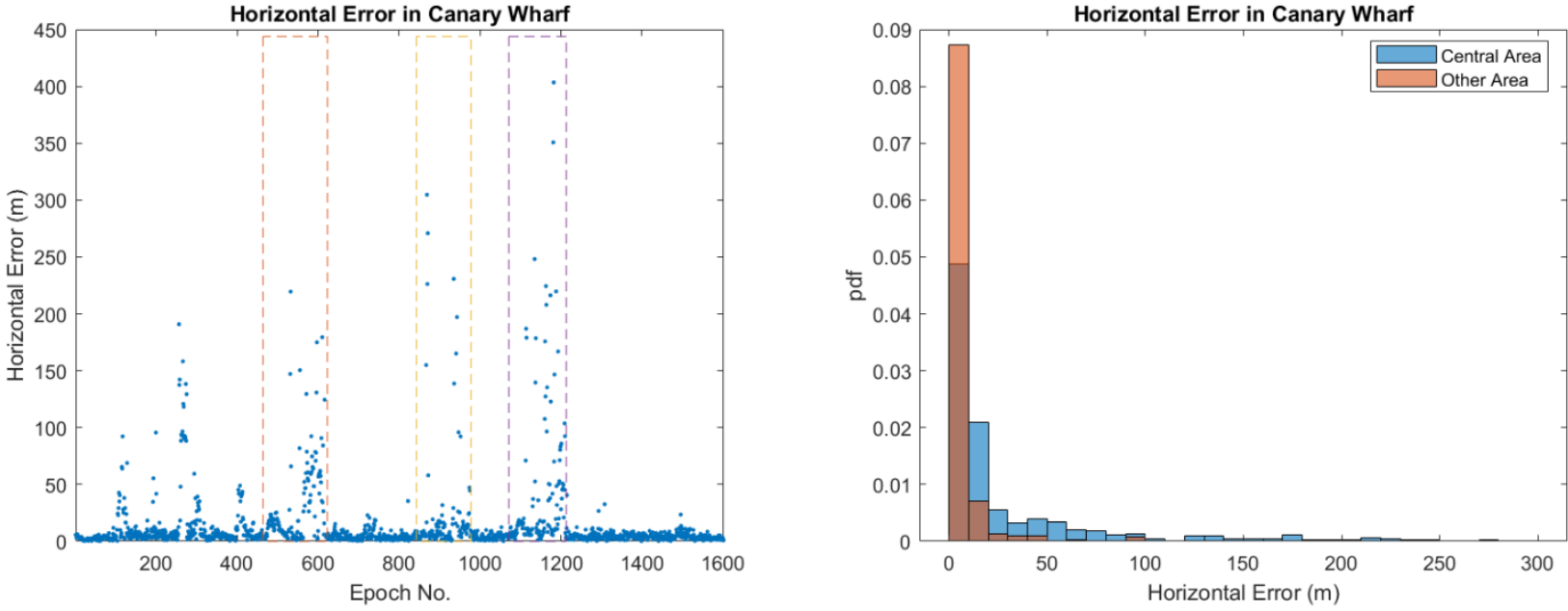

Figure 10: Horizontal radial position error using HDI 3DMA GNSS with conventional GNSS measurements
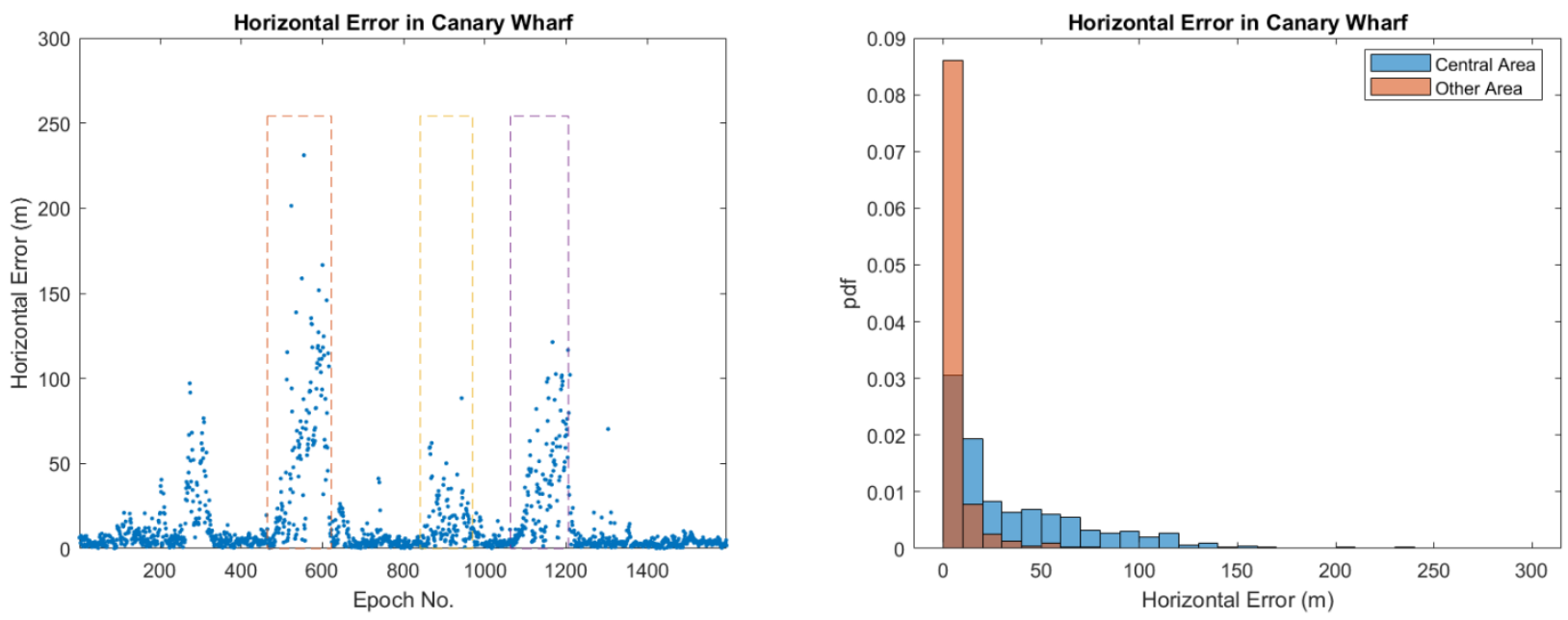

Figure 11: Horizontal radial position error using least-squares ranging with basic S-GNSS measurements 

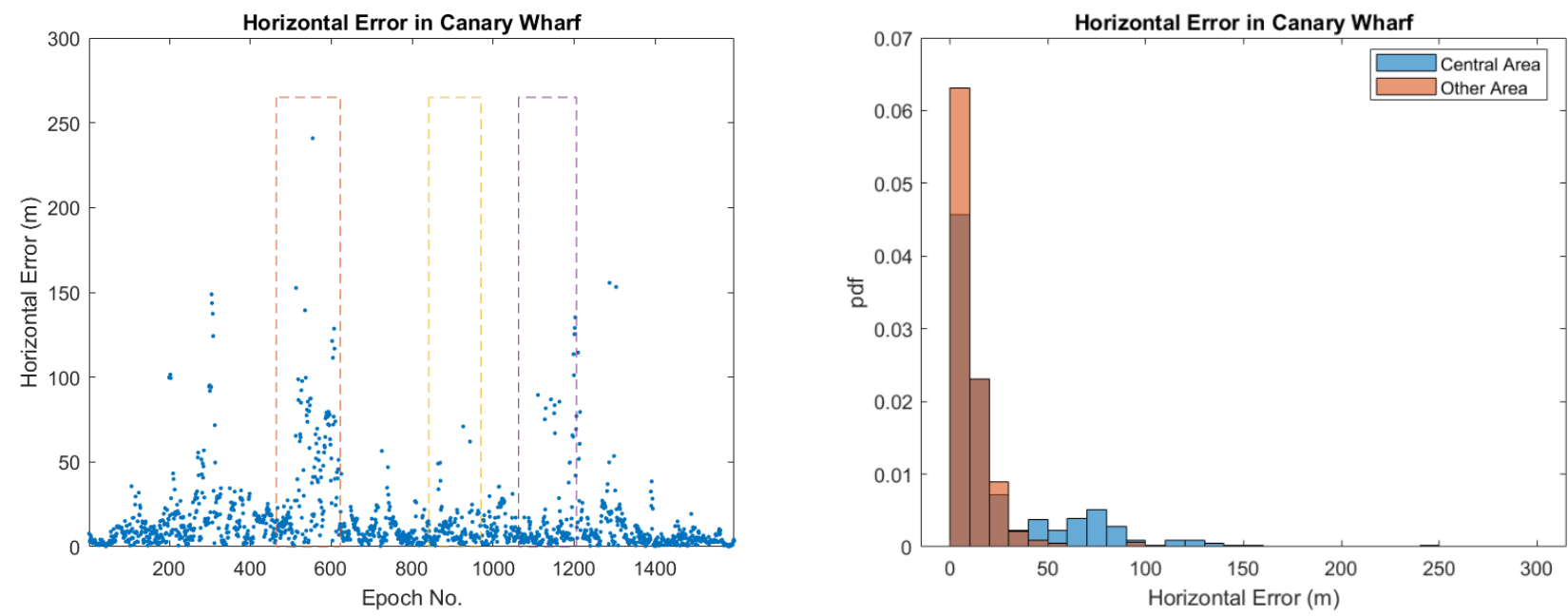

Figure 12: Horizontal radial position error using HDI 3DMA GNSS with basic S-GNSS measurements
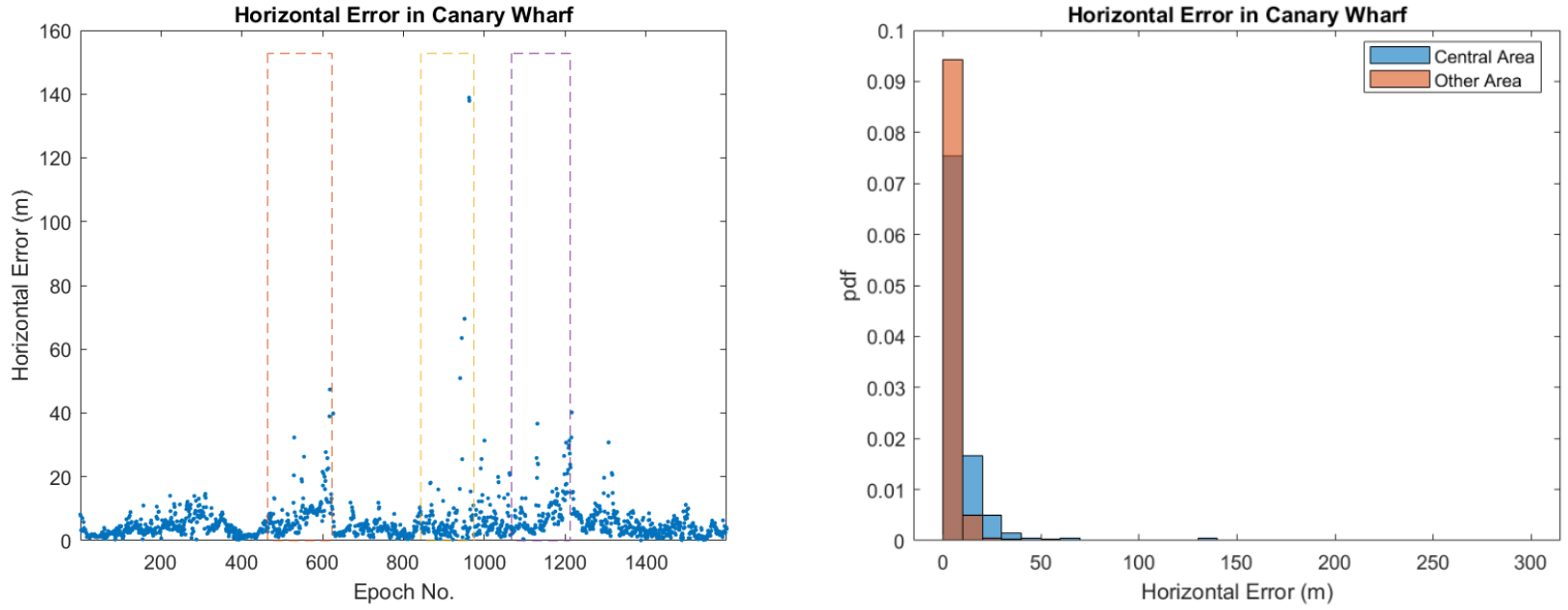

Figure 13: Horizontal radial position error using least-squares ranging with filtered S-GNSS measurements
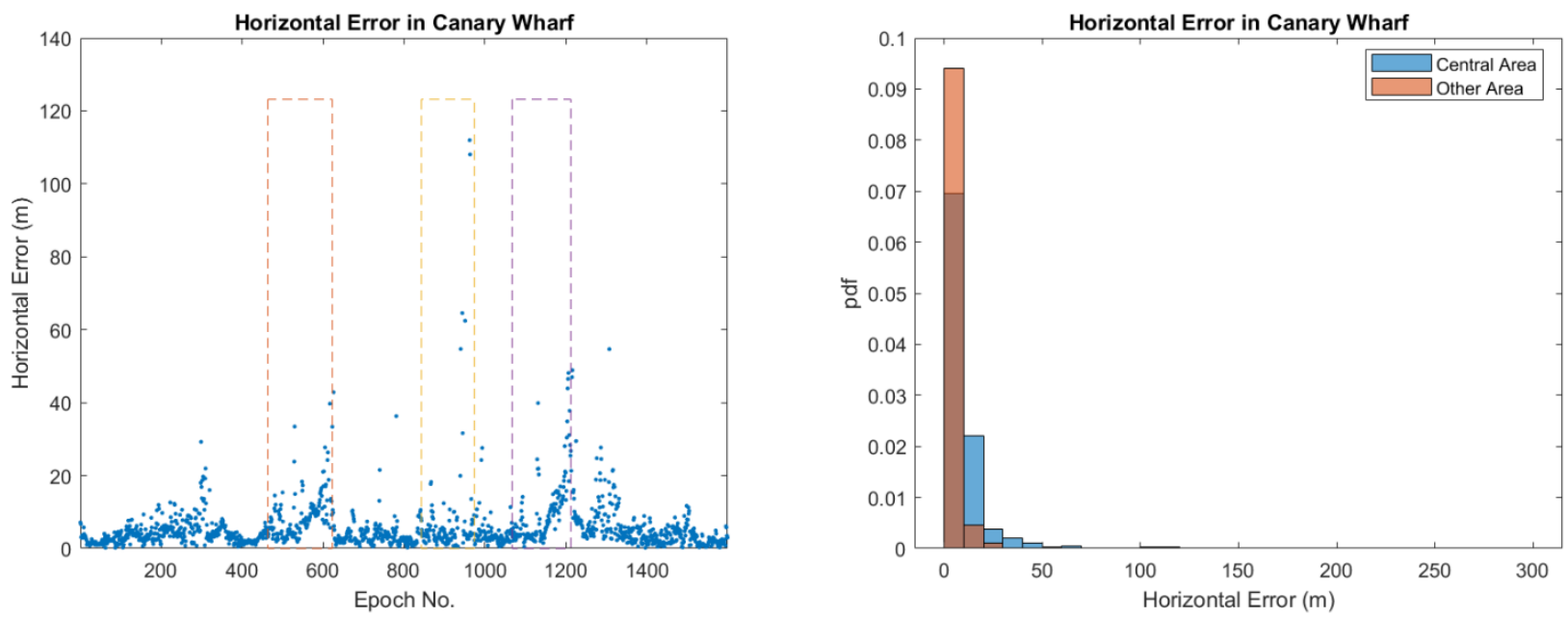

Figure 14: Horizontal radial position error using HDI 3DMA GNSS with filtered S-GNSS measurements

At individual locations, 3DMA GNSS improves positioning accuracy in some cases and not others. Two case studies are included in Appendix B. Further investigation of selected individual results is needed to understand why this is. Different reasons are likely to apply in different places. Mapping errors will sometimes lead to 3DMA GNSS positioning errors as will large passing vehicles that block and reflect GNSS signals. The LSR algorithm currently includes outlier detection while the 3DMA GNSS 
algorithms do not; adding this should help to reduce the impact of vehicles and mapping errors. Also, Lastly, in open areas where conventional GNSS positioning works well, 3DMA GNSS can't be expected to improve on it.

\section{CONCLUSIONS}

The single-epoch positioning results have clearly demonstrated the benefit of supercorrelation with position errors using S-GNSS measurements. Using conventional positioning techniques, using supercorrelation on its own reduces position errors by $40 \%$ using conventional positioning techniques. However, combining supercorrelation and filtered carrier-smoothed pseudo-ranges using outlier detection to reject code components that are inconsistent with the carrier-based component reduces the position errors by a factor of 5.7 in the densest of environments and a factor of 3.5 elsewhere.

For single-epoch positioning, using pseudo-ranges from code tracking only with both conventional and supercorrelation, 3DMA GNSS improves the position accuracy by about $25 \%$ in the denser environments, but does not bring any benefits in the more open areas. However when filtering with outlier detection is used to produce carrier-smoothed pseudo-ranges, 3DMA GNSS techniques have little impact on the positioning accuracy with S-GNSS processing because the impact of NLOS reception has already been reduced. Thus, 3DMA GNSS techniques are likely to be more useful for snapshot positioning techniques and potentially for the initialization of filtered solutions than for continuous positioning.

\section{FUTURE WORK}

There is clearly a need for substantial improvement in the 3DMA GNSS algorithms before this approach can be recommended for use. Areas to investigate include

- Improved scoring models for LBR and SM that take into account additional factors, such as satellite elevation, street width, building height and relative azimuth of the satellite and street direction.

- Optimizing the satellite visibility prediction.

- Finding the best way of combining the LBR and SM likelihood distributions to get an optimal overall positioning solution.

- Outlier detection.

- Improved search area determination, potentially considering non-circular search areas.

- The impact of higher-resolution 3D mapping.

- Multi-epoch 3DMA positioning using Bayesian filtering techniques.

In addition, using S-GNSS Skyscan data to estimate the signal strength, Doppler shift, and pseudo-ranges for all of the different paths the signal takes between the satellite and receiver antennas for each epoch, increases the information per measurement epoch that can be combined with knowledge of the 3D building model

\section{ACKNOWLEDGEMENTS}

Qiming Zhong is funded by the China Scholarship Council and a UCL Engineering Faculty Scholarship. The authors thank UCL $\mathrm{PhD}$ student Eleftherios Plakidis for producing figures 3, 4 and 5 and for assisting with the data collection. The authors also thank Imperial College London for use of their trials van and reference system, and Canary Wharf Group plc, particularly Ms Lesley Johnson, Canary Wharf Central Records Manager, for their permission to collect GNSS data in the Canary Wharf Estate.

\section{APPENDIX A: DETAILED DESCRIPTION OF POSITIONING ALGORITHMS}

This appendix provides more details of the positioning algorithms used to generate the results presented in the paper. Due to the limited duration of the dataset, the same data was used for both tuning and testing of the algorithms. In general, it is better to separate the tuning and testing data.

\section{A.1 LEAST-SQUARES RANGING}

A position solution may be computed from a set of pseudo-range measurements using least-squares estimation. This is given by [10][36]

$$
\hat{\mathbf{x}}^{+}=\hat{\mathbf{x}}^{-}+\left(\mathbf{H}_{G}^{e^{\mathrm{T}}} \mathbf{W}_{\rho} \mathbf{H}_{G}^{e}\right)^{-1} \mathbf{H}_{G}^{e^{\mathrm{T}}} \mathbf{W}_{\rho}\left(\tilde{\mathbf{z}}-\hat{\mathbf{z}}^{-}\right)
$$

where $\hat{\mathbf{x}}^{+}$is the estimated state vector, comprising the position and time solution, $\hat{\mathbf{x}}^{-}$is the predicted state vector, $\tilde{\mathbf{z}}$ is the measurement vector, $\hat{\mathbf{z}}^{-}$is the vector of measurement predictions from $\hat{\mathbf{x}}^{-}, \mathbf{W}_{\rho}$ is the weighting matrix and $\mathbf{H}_{G}^{e}$ is the measurement matrix. Assuming the interconstellation timing bias is known, the state and measurement vectors are given by 


$$
\mathbf{x}=\left(\begin{array}{c}
\mathbf{r}_{e a}^{e} \\
\delta \rho_{c}^{a}
\end{array}\right) \quad \mathbf{z}=\left(\begin{array}{c}
\rho_{a, R}^{1} \\
\rho_{a, R}^{2} \\
\vdots \\
\rho_{a, R}^{m}
\end{array}\right),
$$

where $\mathbf{r}_{e a}^{e}$ is the Cartesian position, resolved about and with respect to an Earth-centred Earth-fixed (ECEF frame), $\delta \rho_{c}^{a}$ is the receiver clock offset, expressed as a range, $\rho_{a, R}^{j}$ is the raw pseudo-range from satellite $j$ and $m$ is the number of satellite used. The measurement matrix is given by

$$
\mathbf{H}_{G}^{e}=\left(\begin{array}{cccc}
-u_{a 1, x}^{e} & -u_{a 1, y}^{e} & -u_{a 1, z}^{e} & 1 \\
-u_{a 2, x}^{e} & -u_{a 2, y}^{e} & -u_{a 2, z}^{e} & 1 \\
\vdots & \vdots & \vdots & \vdots \\
-u_{a m, x}^{e} & -u_{a m, y}^{e} & -u_{a m, z}^{e} & 1
\end{array}\right),
$$

where $\mathbf{u}_{a j}^{e}$ is the line-of-sight vector from the user antenna to satellite $j$, given by

$$
\mathbf{u}_{a s}^{e} \approx\left(\hat{\mathbf{r}}_{e j}^{e}-\hat{\mathbf{r}}_{e a}^{e-}\right) / \hat{r}_{a j}^{-} \quad \hat{r}_{a j}^{-}=\left|\hat{\mathbf{r}}_{e j}^{e}-\hat{\mathbf{r}}_{e a}^{e-}\right|,
$$

where $\hat{\mathbf{r}}_{e j}^{e}$ is the position of satellite $j$ and $\hat{\mathbf{r}}_{e a}^{e-}$ is the predicted user position. The predicted raw pseudo-ranges, used to generate $\hat{\mathbf{z}}^{-}$, are given by

$$
\hat{\rho}_{a, R}^{j-}=\sqrt{\left[\mathbf{C}_{e}^{I}\left(\hat{r}_{a j}^{-}\right) \hat{\mathbf{r}}_{e j}^{e}-\hat{\mathbf{r}}_{e a}^{e-}\right]^{\mathrm{T}}\left[\mathbf{C}_{e}^{I}\left(\hat{r}_{a j}^{-}\right) \hat{\mathbf{r}}_{e j}^{e}-\hat{\mathbf{r}}_{e a}^{e-}\right]}-\delta \hat{\rho}_{c}^{j-}+\delta \hat{\rho}_{\text {trop }}^{j-}+\delta \hat{\rho}_{i o n}^{j-}+\delta \hat{\rho}_{c}^{a-},
$$

where ${ }_{c}^{j}$ is the predicted satellite clock offset including the relativistic clock correction, $\stackrel{{ }^{j}}{j}$ trop is the modeled tropospheric delay, $\quad \hat{i o n}^{j}$ is the modeled ionospheric delay,$\delta \hat{\rho}_{c}^{a-}$ is the predicted receiver clock offset (set to zero here) and $\mathbf{C}_{e}^{I}$ is the Sagnac effect compensation matrix, given by

$$
\mathbf{C}_{e}^{I}\left(r_{a j}\right) \approx\left(\begin{array}{ccc}
1 & \omega_{i e} r_{a j} / c & 0 \\
-\omega_{i e} r_{a j} / c & 1 & 0 \\
0 & 0 & 1
\end{array}\right),
$$

where $\omega_{i e}$ is the Earth rotation rate and noting that (5) and (6) are solved iteratively.

The weighting matrix is given by

$$
\mathbf{W}_{\rho}=\left(\begin{array}{cccc}
\sigma_{\rho 1}^{-2} & 0 & \cdots & 0 \\
0 & \sigma_{\rho 2}^{-2} & \cdots & 0 \\
\vdots & \vdots & \ddots & \vdots \\
0 & 0 & \cdots & \sigma_{\rho m}^{-2}
\end{array}\right)
$$

where

$$
\sigma_{\rho j}=\sqrt{a \times 10^{-\left(C / N_{0}\right)_{j} / 10}},
$$

where $\left(C / N_{0}\right)_{j}$ is the measured carrier-power-to-noise-density ratio of the $j^{\text {th }}$ satellite signal in $\mathrm{dB}-\mathrm{Hz}$ and $a=1.1 \times 10^{4} \mathrm{~m}^{2}$ is a constant.

A Leave One Out approach [40] is used for outlier detection and elimination. The observation vector is assumed to be composed of $m$ completely uncorrelated elements, and the number of unknowns in the state vector is $n=4$. Assuming that there is an outlier in the measurements, the solution calculated from the remaining observations is used to estimate the suspected outlier. The difference between this estimated value and the actual value is then fed to a hypothesis test of an F distribution with 1 degree of freedom in the numerator and $m-n-1$ degrees of freedom in the denominator. The confidence level is set to 0.99 . Where all measurements pass the F-tests, the position solution is accepted. Otherwise, the measurement with the largest difference is then eliminated as it is least consistent with the others and a new least-squares solution calculated. The process repeats until either all observations pass the hypothesis tests or only 4 measurements remain. 
The terrain height is obtained from an Ordnance Survey DTM with a $5 \mathrm{~m}$ horizontal resolution by taking the orthometric height from the database at the nearest 4 points to the least-squares position solution and performing a linear interpolation. The height of the receiver from the ground was set to $1.5 \mathrm{~m}$. The sum of terrain and receiver height was then converted to a geodetic height and added to the geocentric Earth radius to obtain a virtual range measurement, which was added to the measurement vector, $\mathbf{z}$, as the $m+1^{\text {th }}$ component. Where the height from the DTM is used to compute the predicted user position, $\hat{\mathbf{r}}_{e a}^{e-}$, the $m+1^{\text {th }}$ component of the measurement innovation will be zero. The $m+1^{\text {th }}$ row of the measurement matrix will be

$$
\mathbf{H}_{G, m+1}^{e}=\left(\begin{array}{llll}
u_{e a, x}^{e} & u_{e a, y}^{e} & u_{e a, z}^{e} & 0
\end{array}\right),
$$

where $\mathbf{u}_{e a}^{e}$ is the unit vector describing the direction from the center of the Earth to the predicted user position, given by

$$
\mathbf{u}_{e a}^{e} \approx \hat{\mathbf{r}}_{e a}^{e-} /\left|\hat{\mathbf{r}}_{e a}^{e-}\right| \text {. }
$$

The variance of the height-aiding measurement, forming the inverse of the $m+1^{\text {th }}$ diagonal element of $\mathbf{W}_{\rho}$, was assumed to be $10 \mathrm{~m}^{2}$ for the results presented here.

\section{A.2 SEARCH AREA DETERMINATION}

To determine the search area for shadow matching and likelihood-based ranging, a test statistic based on the residuals of the height-aided least-squares ranging solution is used:

$$
s=\sqrt{\frac{\delta \mathbf{z}^{+\mathrm{T}} \delta \mathbf{z}^{+}}{m^{\prime}}} .
$$

where $m^{\prime}$ is the number of measurements after removal of outliers. The test statistic is then used to determine the search area radius and candidate position grid spacing, as shown in Table 2.

Table 2. Search area and grid spacing

\begin{tabular}{|c|c|c|}
\hline Test statistic & Search radius & Grid spacing \\
\hline$s \leq 15 \mathrm{~m}$ & $40 \mathrm{~m}$ & $1 \mathrm{~m}$ \\
\hline$s>15 \mathrm{~m}$ & $200 \mathrm{~m}$ & $5 \mathrm{~m}$ \\
\hline
\end{tabular}

The horizontal error of the least squares ranging solution using the filtered S-GNSS measurements is mostly within 40m. Therefore, for these S-GNSS measurements, the search radius and grid spacing are determined to be $40 \mathrm{~m}$ and $1 \mathrm{~m}$, respectively.

\section{A.3 SHADOW MATCHING}

Once a grid if candidate positions has been set up, shadow matching comprises the following steps [29]:

1. For each candidate position, the probability that the satellite is predicted to be direct line-of-sight, $p(\operatorname{LOS} \mid B B)$, is determined using the building boundaries precomputed from the 3D city model. $p(\operatorname{LOS} \mid B B)$ is set to 0.8 where the satellite elevation is above the building boundary and 0.2 otherwise. These values account for the possibility that predictions may be wrong due to errors and resolution limitations in the 3D city model and unpredictable factors such as passing vehicles.

2. The probability, $p\left(\operatorname{LOS} \mid C / N_{0}\right)$, that each received signal is direct LOS is determined from the GNSS receiver's $C / N_{0}$ measurements using the following statistical model:

$$
p\left(\operatorname{LOS} \mid C / N_{0}\right)=\left\{\begin{array}{cc}
p_{\mathrm{o}-\min } & \left(C / N_{0}\right)<s_{\min } \\
a_{0}+a_{1}\left(C / N_{0}\right)+a_{2}\left[\left(C / N_{0}\right)\right]^{2} & s_{\min }<\left(C / N_{0}\right)<s_{\max } \\
p_{\mathrm{o}-\max } & s_{\max }<\left(C / N_{0}\right)
\end{array} .\right.
$$

where the coefficients are given by Table 3

3. Each candidate position and satellite is scored according to the match between the predicted and measured satellite visibility.

$$
P_{m}=1-p\left(\operatorname{LOS} \mid C / N_{0}\right)-p(\operatorname{LOS} \mid B B)+2 p\left(\operatorname{LOS} \mid C / N_{0}\right) p(\operatorname{LOS} \mid B B) \text {. }
$$

4. An overall likelihood score, $\Lambda_{S p}$, for each position, $p$, is obtained by multiplying the scores for each satellite.

Table 3. Tuning parameters for determining LOS probability from measured $C / N_{0}$

\begin{tabular}{|c|c|c|c|c|c|c|c|c|}
\hline Elevation, $\theta$ & Satellites & $s_{\min }, \mathrm{dB}-\mathrm{Hz}$ & $s_{\max }, \mathrm{dB}-\mathrm{Hz}$ & $p_{\min }, \mathrm{dB}-\mathrm{Hz}$ & $a_{0}, \mathrm{~dB}-\mathrm{Hz}$ & $a_{1}, \mathrm{~dB}-\mathrm{Hz}$ & $a_{2}, \mathrm{~dB}-\mathrm{Hz}$ & $p_{\max }, \mathrm{dB}-\mathrm{Hz}$ \\
\hline$\theta \leq 20^{\circ}$ & All & 16 & 32 & 0.2 & -0.417 & 0.03735 & 0 & 0.8 \\
\hline $20^{\circ} \leq \theta \leq 60^{\circ}$ & GPS & 26 & 40 & 0.2 & -0.8369 & 0.04062 & 0 & 0.8 \\
\hline $20^{\circ} \leq \theta \leq 60^{\circ}$ & Galileo & 21 & 34 & 0.2 & 0.6333 & -0.06324 & 0.002019 & 0.8 \\
\hline $60^{\circ} \leq \theta$ & All & 33 & 40 & 0.2 & -2.785 & 0.08968 & 0 & 0.8 \\
\hline
\end{tabular}




\section{A.4. LIKELIHOOD-BASED RANGING}

Once a grid if candidate positions has been set up, the likelihood-based 3DMA ranging algorithm, comprising the following steps [37]:

1. For each candidate position, predict the satellite visibility using the building boundaries.

2. At each candidate position, the satellites are scored based on the elevation angle, measured $C / N_{0}$ and surrounding buildings, and the one with the highest score is selected as the reference. The score is calculated by

$$
\text { score }_{j}=\frac{1}{n} \sum_{p=1}^{n}\left(\text { elev }_{j}-B B_{p}\right) \times \text { round }\left(\left(C / N_{0}\right)_{j} / 5\right) \times 5 \text {. }
$$

where $\frac{1}{n} \sum_{p=1}^{n}\left(e l e v_{j}-B B_{p}\right)$ is the average value of the difference between the satellite elevation, elev $v_{j}$, and the corresponding building boundary, $B B_{p}$, at the candidate point, $p=1$, and its immediate neighbors, $p=2,3, \cdots, n$.

3. At each candidate position, the direct LOS range to each satellite is computed as described in Appendix A.1. Measurement innovations are obtained by subtracting the computed ranges from the measured pseudo-ranges and then differencing with respect to the reference satellite.

4. The error standard deviation of all errors except for the NLOS path delay is computed as a function of $C / N_{0}$ using.

$$
\sigma_{j}=\sqrt{a \times 10^{-\left(C / N_{0}\right)_{j} / 10}+b} .
$$

where $a$ and $b$ are empirically determined constant. The mean and standard deviation of the NLOS path delay, $\mu_{N}$ and $\sigma_{N}$, are listed in the Table 4.

5. At each candidate position, the measurement innovation for each satellite predicted to be LOS is modeled by a normal distribution with a mean of $\mu_{L}$. The NLOS innovation is re-mapped by using a skew normal distribution to determine the cumulative probability and then substituting the corresponding direct LOS innovation with the same cumulative probability. First of all parameters describing the skew-normal distribution are computed:

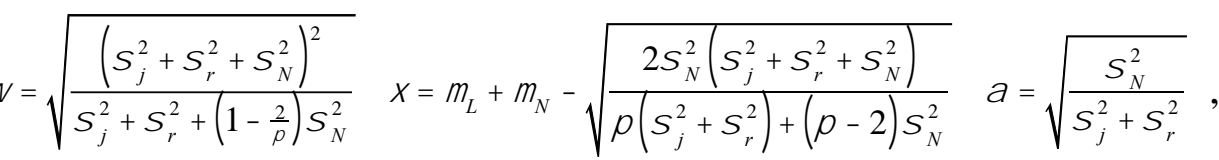

where $\sigma_{r}$ is the error standard deviation of the reference satellite, which is a constant. The cumulative probability, F, of the NLOS measurement innovation, $\delta z_{p j}$, is then computed using

$$
F=\frac{1}{2}\left[1+\operatorname{erf}\left(\frac{z_{p j}}{\sqrt{2}}\right)\right] 2 T\left(\frac{z_{p j}}{},\right),
$$

where erf is the integral of the normal distribution and $T$ is Owen's $\mathrm{T}$ function. These are given by

$$
\operatorname{erf}(x)=\frac{2}{\sqrt{\pi}} \int_{0}^{x} \exp \left(-t^{2}\right) d t \quad T(x, \alpha)=\frac{1}{2 \pi} \int_{0}^{\alpha} \frac{\exp \left(-\frac{1}{2} x^{2}\left(1+t^{2}\right)\right)}{1+t^{2}} d t,
$$

and can be calculated using open-source software. Finally, a modified measurement innovation, $\delta z_{p j}^{\prime}$, is obtained from $F$ by solving

$$
F=\frac{1}{2}\left[1+\operatorname{erf}\left(\frac{\delta z_{p j}^{\prime}-\mu_{L}}{\sqrt{2\left(\sigma_{j}^{2}+\sigma_{r}^{2}\right)}}\right)\right] .
$$

Again, open-source software can be used. For measurements predicted to be direct LOS, $\delta z_{p j}^{\prime}=\delta z_{p j}$.

6. To prevent excessively large innovation producing very low likelihood scores, limiting is applied to each innovation

$$
z_{p j}=\max \left(z_{\max }, \min \left(z_{\max }, z_{p j} \quad L\right)\right) .
$$

where $\delta z_{\max }=22 \mathrm{~m}$ in the conventional dataset and $\delta z_{\max }=18.5 \mathrm{~m}$ in the basic and filtered S-GNSS dataset, based on 3 standard deviations for the case where $\sigma_{j}=5.2 \mathrm{~m}$ and $\sigma_{j}=4.4 \mathrm{~m}$, respectively.

7. A likelihood score for each candidate position, $p$, is then computed using

$$
\Lambda_{R p}=\exp \left(-\delta \mathbf{z}_{p}^{\prime \mathrm{T}} \mathbf{C}_{\delta \mathbf{z}, p}^{-1} \delta \mathbf{z}_{p}^{\prime}\right),
$$


where $\delta \mathbf{z}_{p}^{\prime}$ is the vector of re-mapped measurement innovations and $\mathbf{C}_{\delta \mathbf{z}, p}$ is the measurement error covariance matrix, given by.

$$
\mathbf{C}_{\delta \boldsymbol{L}}=\left(\begin{array}{cccc}
\sigma_{1}^{2}+\sigma_{r}^{2} & \sigma_{r}^{2} & \cdots & \sigma_{r}^{2} \\
\sigma_{r}^{2} & \sigma_{2}^{2}+\sigma_{r}^{2} & \cdots & \sigma_{r}^{2} \\
\vdots & \vdots & \ddots & \vdots \\
\sigma_{r}^{2} & \sigma_{r}^{2} & \cdots & \sigma_{m}^{2}+\sigma_{r}^{2}
\end{array}\right),
$$

noting that the reference satellite ranging error is correlated across all measurement innovations.

Table 4. Parameters used in the likelihood-based ranging (LBR)

\begin{tabular}{|c|c|c|c|c|c|c|}
\hline Dataset & $\mathrm{a}, \mathrm{m}^{2}$ & $\mathrm{~b}, \mathrm{~m}^{2}$ & $\mu_{N}, \mathrm{~m}$ & $\sigma_{N}, \mathrm{~m}$ & $\mu_{L}, \mathrm{~m}$ & $\sigma_{r}, \mathrm{~m}$ \\
\hline Conventional & $1.41 \times 10^{4}$ & 28.1 & 26.06 & 31.76 & -5.25 & 2.36 \\
\hline Basic S-GNSS & 5751 & 40.388 & 9.533 & 16.48 & -6.051 & 3.15 \\
\hline Filtered S-GNSS & 2091 & 13.68 & 0.5 & 4.36 & -4.935 & 4.4 \\
\hline
\end{tabular}

\section{A.5. HYPOTHESIS-DOMAIN INTEGRATION}

Hypothesis-domain integration combines the shadow-matching and 3DMA ranging scores to give a single score for each candidate position:

$$
\tilde{\Lambda}_{p}=\Lambda_{R p} \Lambda_{S p}^{W_{p}} \quad \forall p,
$$

where $W_{p}$ is the shadow-matching weighting factor, which is a constant of 2.9 in the filtered S-GNSS. But in the conventional and the basic S-GNSS datasets, it is a value related to the number of LOS signals in the current epoch.

$$
W_{p}=\frac{\alpha \times n_{L O S}}{n_{L O S}+n_{N L O S}},
$$

where is an empirical constant. Finally, the position solution is obtained using

$$
\hat{E}_{a}=\frac{\sum_{p} \Lambda_{p} E_{p}}{\sum_{p} \Lambda_{p}}, \quad \hat{N}_{a}=\frac{\sum_{p} \Lambda_{p} N_{p}}{\sum_{p} \Lambda_{p}},
$$

where $E_{p}$ and $N_{p}$ are the easting and northing coordinates of the $p^{\text {th }}$ candidate position.

\section{APPENDIX B: CASE STUDIES}

Figures 15 and 16 show scoring maps from likelihood-based ranging (LBR) and shadow matching (SM) at points P1 and P2, respectively. The location of these is shown in Figure 17. P1 is in the high-density central area while P2 is in a more open area. In both cases, the shadow-matching solution includes a high-scoring area on the correct side of the street close to the true position. However, both show an additional high-scoring area at an incorrect location. Because of this solution ambiguity, simply taking a weighted average of the candidate positions leads to a relatively poor position solution. At P2 (figure 16), it can be seen that the zero scores for the indoor candidates effectively push the position solution away from the highest scoring areas, in this case actually bringing it closer to the truth.

At P2 (figure 16), the LBR algorithm provides a good position solution a few meters away from the truth. However, the highscoring areas of the SM and LBR likelihood distributions do not overlap, so multiplying both distributions together leads to an ambiguous solution with multiple peaks in the combined likelihood surface. At P1 (figure 15), the situation is worse; here, the LBR solution is wrong, with the high-scoring region not matching either the true position or the shadow-matching distribution. Note that the maximum score is much higher for the P2 LBR solution than it is for the P1 solution, so this can potentially be used as a quality indicator, enabling poorer solutions to be rejected in favor of the conventional LSR solution.

Clearly, more work is needed to improve the 3DMA GNSS algorithms, considering the candidate position scoring models for both SM and LBR, the way in which the two distributions are combined to produce an integrated 3DMA GNSS solution, and how a position solution is then extracted from the distribution. 

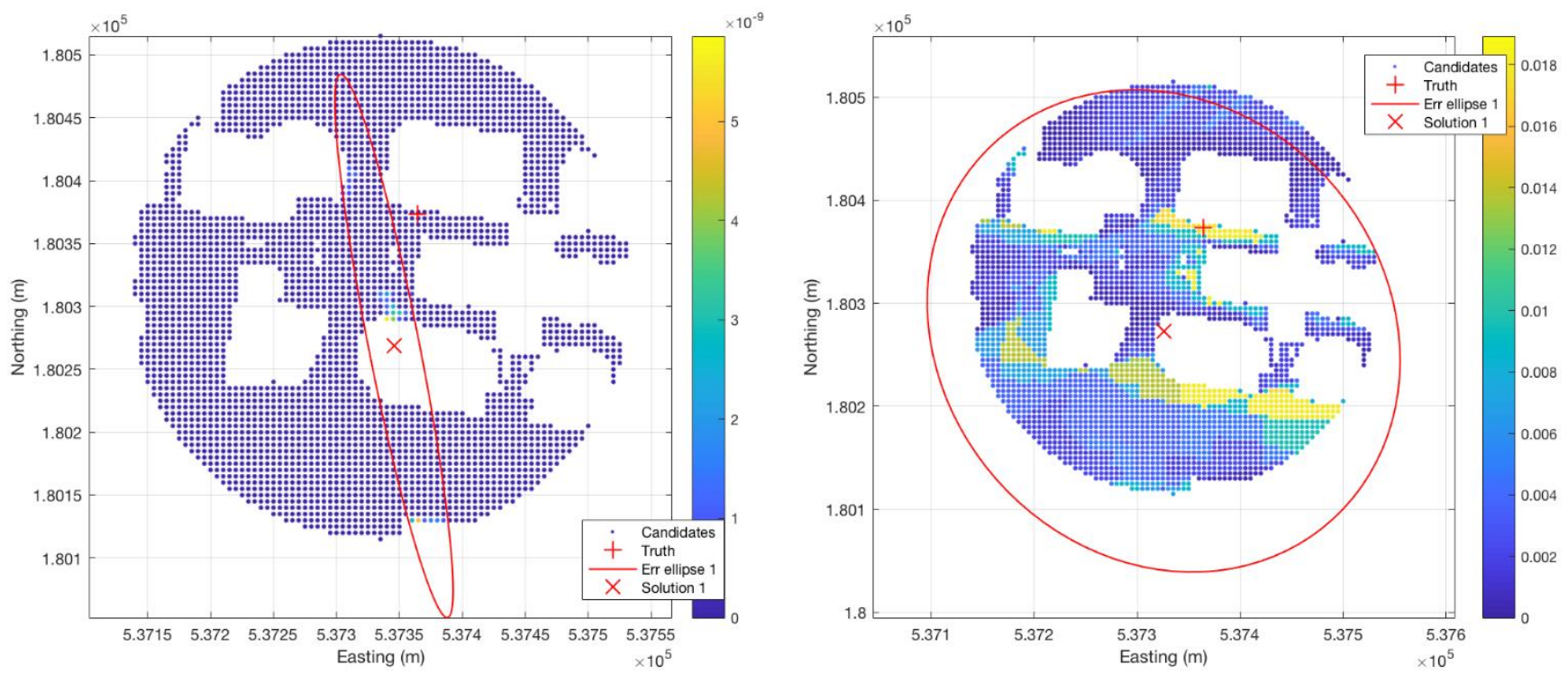

Figure 15: Scoring maps from likelihood-based ranging (LBR, left plot) and shadow matching (SM, right plot) at point P1, epoch 1113 , in a dense urban area (white areas denote buildings)
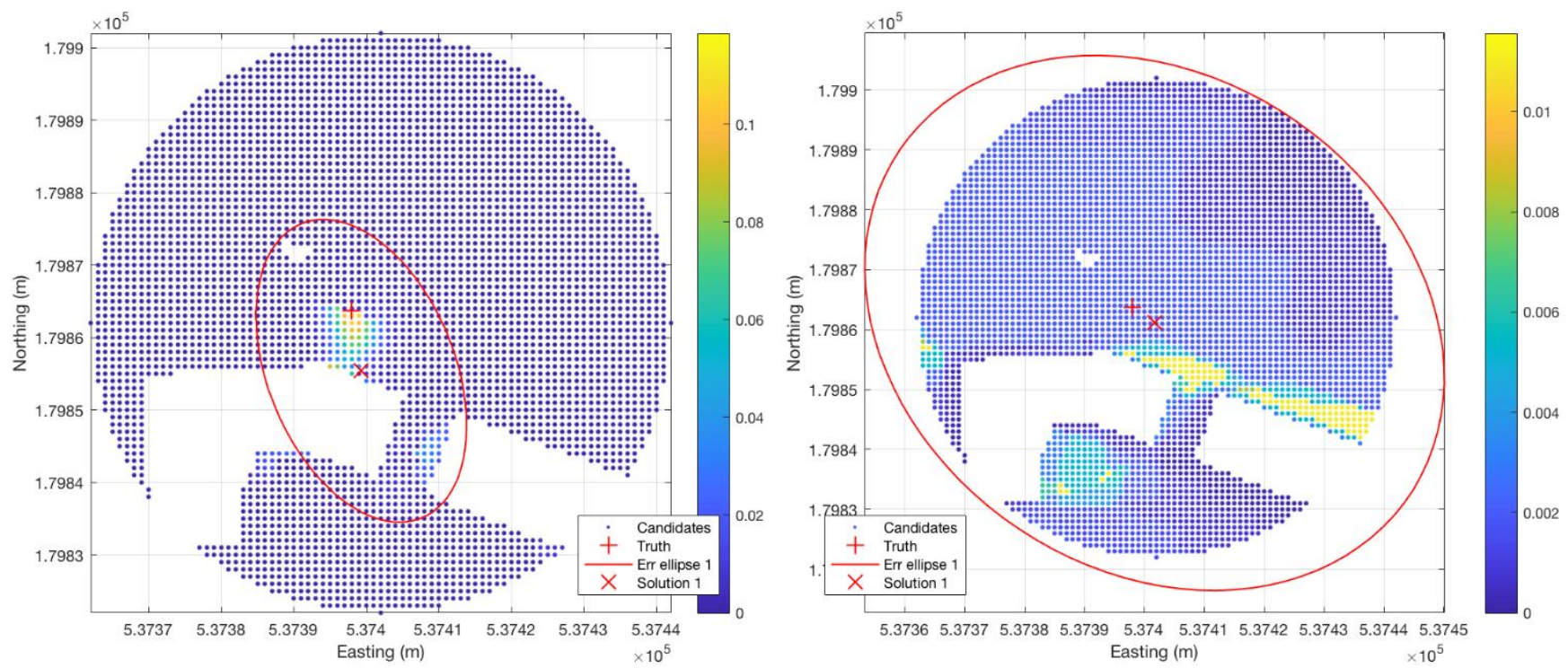

Figure 16: Scoring maps from likelihood-based ranging (LBR, left plot) and shadow matching (SM, right plot) at point P2, epoch 240 , in a relatively open area (white areas denote buildings) 


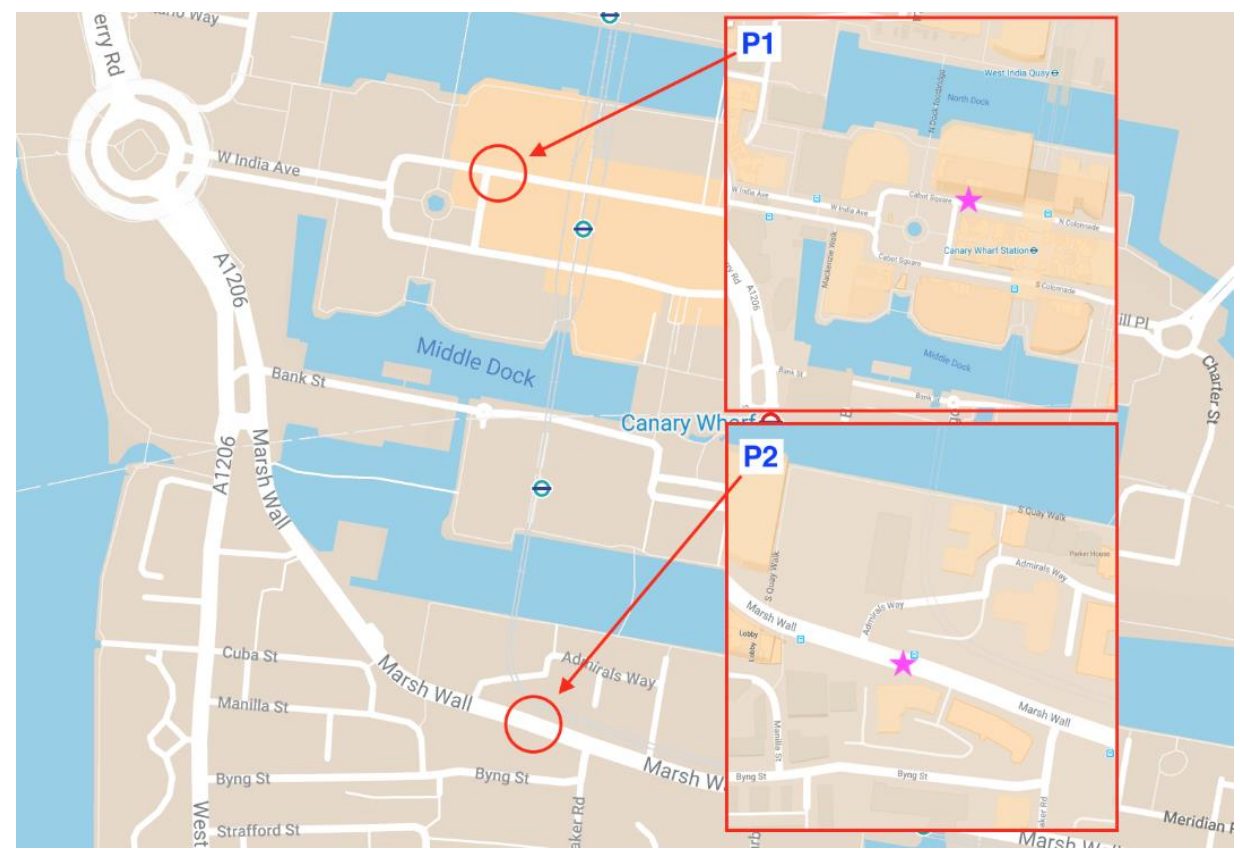

Figure 17: Locations of points P1 and P2. Background map (C) Google

\section{REFERENCES}

[1] Groves, P. D. "GNSS solutions: Multipath vs. NLOS signals. how does Non-Line-of-Sight reception differ from multipath interference". Inside GNSS Magazine, Nov/Dec 2013, pp. 40-42, 63.

[2] Groves, P. D., et al. "A portfolio approach to NLOS and multipath mitigation in dense urban areas". ION GNSS+ 2013, Nashville, TN.

[3] Faragher, R. et al. "Method, Apparatus, Computer Program, Chip Set, or Data Structure for Correlating a Digital Signal and a Correlation Code". U.S. Patent Application No. 15/464,485.

[4] Faragher, R. et al., "Supercorrelation: Enhancing the Accuracy and Sensitivity of Consumer GNSS Receivers with a DSP Upgrade," ION GNSS+ 2018, Miami, FL.

[5] Esteves, P., and Faragher, R., S-GNSS Technical Overview White Paper, Version 1.1., Focal Point Positioning Ltd, July 2020.

[6] Ward, P. W., “GNSS Receivers,” In Kaplan, E. D. and Hegarty, C.J. (Eds), Understanding GPS/GNSS Principles and Applications, Third Edition, Artech House, 2017, pp. 339-548.

[7] Van Diggelen, F., A-GPS: Assisted GPS, GNSS, and SBAS, Artech House, 2009.

[8] Ward, P. W., Betz, J. W., and Hegarty, C., “GNSS Disruptions,” In Kaplan, E. D. and Hegarty, C.J. (Eds), Understanding GPS/GNSS Principles and Applications, Third Edition, Artech House, 2017, pp. 549-617.

[9] McGraw, G. A., Groves, P. D., and Ashman, B. W., "Robust Positioning in the Presence of Multipath and NLOS GNSS Signals," In Morton, J., van Diggelen, F., Spilker, J, Jr, and Parkinson, B. (Eds), Position, Navigation, and Timing Technologies in the 21st Century: Integrated Satellite Navigation,Sensor Systems, and Civil Applications, Wiley-IEEE Press, 2020.

[10] Groves, P. D., Principles of GNSS, inertial, and multi-sensor integrated navigation systems, Second Edition, Artech House, 2013.

[11] Pany, T., Eissfeller, B., "Demonstration of a Synthetic Phased Array Antenna for Carrier/Code Multipath Mitigation," ION GNSS 2008, Savannah, GA, pp. 663-668.

[12] Soloviev, A., and F. Van Graas, "Beam Steering in Global Positioning System Receivers using Synthetic Phased Arrays," IEEE Transactions of Aerospace and Electronic Systems, Vol. 446, No. 3, 2010, pp. 1513-1521.

[13] Raquet, J. F., "What's Next for Practical Ubiquitous Navigation: World Models and Magnetic Field Maps," Inside GNSS Sept/Oct 2013.

[14] Obst, M., S. Bauer, and G. Wanielik, "Urban Multipath Detection and mitigation with Dynamic 3D Maps for Reliable Land Vehicle Localization," IEEE/ION PLANS 2012.

[15] Peyraud, S., et al., "About Non-Line-Of-Sight Satellite Detection and Exclusion in a 3D Map-Aided Localization Algorithm," Sensors, Vol. 13, 2013, pp. 829-847.

[16] Suzuki, T., "Integration of GNSS Positioning and 3D Map using Particle Filter" ION GNSS+ 2016, Portland, Oregon.

[17] Suzuki, T., and Kubo N., "Correcting GNSS Multipath Errors Using a 3D Surface Model and Particle Filter," ION GNSS+ 2013, Nashville, TN. 
[18] Hsu, L.-T., Gu, Y., and Kamijo, S., “3D building model-based pedestrian positioning method using GPS/GLOANSS/QZSS and its reliability calculation”, GPS Solutions, 20(3), 2016, 413-428.

[19] Ziedan, N I., "Urban Positioning Accuracy Enhancement Utilizing 3D Buildings Model and Accelerated Ray Tracing Algorithm," ION GNSS+ 2017, Portland, OR.

[20] Liu, X., Nath, S., and Govindan, R.. “ Gnome: A Practical Approach to NLOS Mitigation for GPS Positioning in Smartphones,". In MobiSys '18: The 16th Annual International Conference on Mobile Systems, Applications, and Services, June 10-15, 2018, Munich, Germany. ACM.

[21] Hsu, L.-T., "Analysis and modeling GPS NLOS effect in highly urbanized area". GPS Solutions, Vol. 22, No. 1, 20187, Article 7.

[22] Ng, H.-F., Zhang, G., and Hsu, L.-T., "Range-based 3D Mapping Aided GNSS with NLOS Correction based on Skyplot with Building Boundaries," ION Pacific PNT, Honolulu, Hawaii, 2019.

[23] Kumar, R. and Petovello, M, G., "A Novel GNSS Positioning Technique for Improved Accuracy in Urban Canyon Scenarios using 3D City Model", ION GNSS+ 2014, Tampa, FL.

[24] Groves, P. D., "Shadow Matching: A New GNSS Positioning Technique for Urban Canyons". Journal of Navigation, 64(3), 2011, 417-430. Also available from http://discovery.ucl.ac.uk/.

[25] Ben-Moshe, B., et al., "Improving Accuracy of GNSS Devices in Urban Canyons". 23rd Canadian Conference on Computational Geometry, 2011.

[26] Wang, L., Groves, P. D. and Ziebart, M. K., “GNSS Shadow Matching Using A 3D Model of London”. European Navigation Conference, London, 2011.

[27] Suzuki, T., and Kubo, N., "GNSS Positioning with Multipath Simulation using 3D Surface Model in Urban Canyon". ION GNSS 2012, Nashville, TN.

[28] Wang, L., Groves, P. D. and Ziebart, M. K., "GNSS Shadow Matching: Improving Urban Positioning Accuracy Using a 3D City Model with Optimized Visibility Prediction Scoring”. NAVIGATION, 60(3), 2013, 195-207. (First published at ION GNSS, 2012, Nashville, TN). Also available from http://discovery.ucl.ac.uk/.

[29] Wang, L., Groves, P. D. and Ziebart, M. K., "Smartphone Shadow Matching for Better Cross-street GNSS Positioning in Urban Environments". Journal of Navigation, 68(3), 2015, 411-433. Also available from http://discovery.ucl.ac.uk/.

[30] Yozevitch, R. and Ben-Moshe, B., “A Robust Shadow Matching Algorithm for GNSS Positioning," NAVIGATION, 62(2), 2015, 95-109.

[31] Irish, A., Iland, D., and Madhow, U., "Urban Localization and 3D Mapping Using GNSS Shadows," Inside GNSS, Sept/Oct 2015.

[32] Groves, P. D., et al., "GNSS Shadow Matching: The Challenges Ahead”. ION GNSS+ 2015, Tampa, Florida, pp. 24212423.

[33] Wang, L., Groves, P. D. and Ziebart, M. K., "Multi-Constellation GNSS Performance Evaluation for Urban Canyons Using Large Virtual Reality City Models," Journal of Navigation, 65(3), 2012, 459-476.

[34] Van Diggelen, F., Want, R., and Wang, W., "One-meter Accuracy in Android,” GPS World, July 2018, pp. 18-34.

[35] Wang, L., Groves, P. D. and Ziebart, M. K., "Urban Positioning on a Smartphone: Real-time Shadow Matching Using GNSS and 3D City Models". ION GNSS+ 2013, Nashville, Tennessee. AND Inside GNSS, Nov/Dec 2013, 44-56.

[36] Groves, P. D. and Jiang, Z., "Height Aiding, C/N0 Weighting and Consistency Checking for GNSS NLOS and Multipath Mitigation in Urban Areas". Journal of Navigation, 66, 653-669 (2013)

[37] Groves, P. D, "Draft Algorithm Specification for Hypothesis-based GNSS Ranging”, UCL internal report, February 2016.

[38] Amt, J. H. R., and Raquet, J. F., "Positioning for Range-Based Land Navigation Systems Using Surface Topography". ION GNSS 2006, Fort Worth, TX.

[39] iMar Navigation and Control, iTraceRT: Accurate INS/GNSS Systems for Automotive Applications, https://www.imarnavigation.de/en/products/by-product-names/item/itracert-f200-itracert-f400-itracert-mvt. Accessed 9/9/2020.

[40] Biagi, L., \& Caldera, S. “An Efficient Leave One Block Out approach to identify outliers”, Journal of Applied Geodesy, 7(1), 2013 11-19. 